\title{
Hfq assists small RNAs in binding to the coding sequence of $o m p D$ mRNA and in rearranging its structure
}

\author{
ZUZANNA WROBLEWSKA and MIKOLAJ OLEJNICZAK \\ Institute of Molecular Biology and Biotechnology, Faculty of Biology, Adam Mickiewicz University in Poznań, 61-614 Poznań, Poland
}

\begin{abstract}
The bacterial protein $\mathrm{Hfq}$ participates in the regulation of translation by small noncoding RNAs (sRNAs). Several mechanisms have been proposed to explain the role of $\mathrm{Hfq}$ in the regulation by sRNAs binding to the $5^{\prime}$-untranslated mRNA regions. However, it remains unknown how $\mathrm{Hfq}$ affects those sRNAs that target the coding sequence. Here, the contribution of $\mathrm{Hfq}$ to the annealing of three sRNAs, RybB, SdsR, and MicC, to the coding sequence of Salmonella ompD mRNA was investigated. Hfq bound to ompD mRNA with tight, subnanomolar affinity. Moreover, Hfq strongly accelerated the rates of annealing of RybB and MicC sRNAs to this mRNA, and it also had a small effect on the annealing of SdsR. The experiments using truncated RNAs revealed that the contributions of $\mathrm{Hfq}$ to the annealing of each sRNA were individually adjusted depending on the structures of interacting RNAs. In agreement with that, the mRNA structure probing revealed different structural contexts of each sRNA binding site. Additionally, the annealing of RybB and MicC sRNAs induced specific conformational changes in ompD mRNA consistent with local unfolding of mRNA secondary structure. Finally, the mutation analysis showed that the long AU-rich sequence in the $5^{\prime}$-untranslated mRNA region served as an Hfq binding site essential for the annealing of sRNAs to the coding sequence. Overall, the data showed that the functional specificity of $\mathrm{Hfq}$ in the annealing of each sRNA to the ompD mRNA coding sequence was determined by the sequence and structure of the interacting RNAs.
\end{abstract}

Keywords: Hfq; sRNA; ribosome; coding sequence; mRNA; ompD

\section{INTRODUCTION}

The Hfq protein is involved in the regulation of translation by bacterial small RNAs (sRNAs) (Updegrove et al. 2016). These noncoding RNAs recognize complementary sequences in their target mRNAs, and induce the activation or repression of translation (Waters and Storz 2009; Updegrove et al. 2015). This regulation is important for the adaptation of enterobacteria to changing environmental conditions (De Lay and Gottesman 2012), maintenance of cellular homeostasis (Papenfort et al. 2013), and virulence of pathogenic species (Papenfort and Vogel 2010; Tree et al. 2014). Hfq is an Sm-like protein, which has a shape of a homohexameric ring (Moller et al. 2002; Zhang et al. 2002). It binds uridine-rich sRNAs using its proximal face (Mikulecky et al. 2004; Sauer and Weichenrieder 2011; Panja et al. 2015) and the outer rim (Sauer et al. 2012), and it binds mRNAs containing $(\mathrm{ARN})_{n}$ sequence motifs using its distal face (de Haseth and Uhlenbeck 1980; Link et al. 2009). However, recent studies have suggested that other modes of RNA interactions with $\mathrm{Hfq}$ are also possible, both in Escherichia coli (Zhang et al. 2013; Małecka et al. 2015; Schu et al.

\footnotetext{
Corresponding author: mol@amu.edu.pl

Article published online ahead of print. Article and publication date are at http://www.rnajournal.org/cgi/doi/10.1261/rna.055251.115. Freely available online through the RNA Open Access option.
}

2015) and in Gram-positive bacteria (Kovach et al. 2014; Robinson et al. 2014).

Hfq participates in sRNA-dependent translation regulation in different ways. The binding of sRNAs by Hfq protects them from degradation (Sledjeski et al. 2001; Moller et al. 2002; Andrade et al. 2012; Fei et al. 2015), but Hfq can also recruit the degradosome to sRNA-mRNA complexes, which leads to their accelerated decay (Ikeda et al. 2011). Moreover, Hfq promotes the pairing of certain sRNAs to their mRNA targets (Moller et al. 2002; Zhang et al. 2002; Maki et al. 2008; Soper and Woodson 2008), and facilitates the annealing of regulatory RNA-OUT to transposase-encoding RNA-IN (Ross et al. 2013). However, other roles of Hfq in translation regulation are also possible. For example, $\mathrm{Hfq}$ directly interferes with $s d h C$ mRNA translation after being recruited by Spot42 sRNA, which binds to this mRNA independently of Hfq (Desnoyers and Masse 2012). Similarly, the recruitment of $\mathrm{Hfq}$ by SgrS sRNA is necessary for purR mRNA translation repression (Bobrovskyy and Vanderpool 2016). In another example, Hfq has been shown to compete with RyhB sRNA for binding to cirA mRNA, which leads to their opposite functions in the regulation (Salvail et al.

(C) 2016 Wroblewska and Olejniczak This article, published in RNA, is available under a Creative Commons License (Attribution 4.0 International), as described at http://creativecommons.org/licenses/by/4.0/. 
2013). Finally, Hfq has been proposed to repress translation independently of sRNAs by direct binding to amiE mRNA (Sonnleitner and Blasi 2014) or to RNA-IN mRNA (Ellis et al. 2015). Overall, these data indicate that Hfq may have different contributions to sRNA stability, their annealing to mRNAs, and translation regulation.

The detailed molecular mechanism used by Hfq has been explained only for its role in the positive regulation of E. coli rpoS mRNA by DsrA sRNA, which targets the $5^{\prime}$-untranslated region of this mRNA (Soper and Woodson 2008; Soper et al. 2011; Peng et al. 2014a,b). Hfq forms multilateral interactions with rpoS mRNA, resulting in a distorted, more compact structure of mRNA, which facilitates the annealing of DsrA to rpoS (Soper et al. 2011; Peng et al. 2014a). The binding of Hfq to an $(\mathrm{ARN})_{4}$ sequence motif in rpoS mRNA, which correctly positions $\mathrm{Hfq}$ in relation to the DsrA binding site, is essential for the DsrA-rpoS pairing (Soper and Woodson 2008; Peng et al. 2014 b). However, it is not known whether a similar mechanism is used by $\mathrm{Hfq}$ to contribute to translation regulation by other sRNAs, especially those which bind to the mRNA coding sequence and exert negative regulation of translation.

Recent Hfq profiling data in a pathogenic E. coli strain showed that almost $40 \%$ of recovered reads mapped to the coding regions (Tree et al. 2014), which suggested that the coding sequence could be an important target of Hfq-dependent regulation. Indeed, although the majority of sRNAs target the $5^{\prime}$-untranslated regions, several Salmonella and E. coli sRNAs bind within the coding sequence of mRNAs (Bouvier et al. 2008; Pfeiffer et al. 2009; Gutierrez et al. 2013; Papenfort et al. 2013; Guo et al. 2014; Bobrovskyy and Vanderpool 2016). Some sRNAs bind just downstream from the start codon and are expected to interfere with the initiation of translation (Bouvier et al. 2008; Balbontin et al. 2010; Papenfort et al. 2010; Guo et al. 2014). The annealing sites of other sRNAs are located downstream from the footprint of the initiation complex (Pfeiffer et al. 2009; Frohlich et al. 2012; Gutierrez et al. 2013; Bobrovskyy and Vanderpool 2016), despite the fact that the elongating ribosome is expected to efficiently unwind helical structures on its path (Takyar et al. 2005; Qu et al. 2011). The coding sequence of Salmonella ompD mRNA contains the binding sites of four sRNAs, which are RybB (Papenfort et al. 2010), SdsR (Frohlich et al. 2012), InvR (Pfeiffer et al. 2007), and MicC (Pfeiffer et al. 2009). Among them RybB sRNA represses the initiation of translation (Papenfort et al. 2010), while MicC induces the accelerated decay of $o m p D$ mRNA, which is dependent on RNase E (Pfeiffer et al. 2009; Bandyra et al. 2012). The coimmunoprecipitation studies showed that ompD mRNA was bound by Hfq (Sittka et al. 2008). Moreover, the OmpD protein expression was increased in $h f q$ deletion strains, which suggested the involvement of Hfq in ompD translation regulation (Sittka et al. 2007; Bossi et al. 2008). However, it is not known how Hfq participates in this regulation.

In order to evaluate the role of Hfq in the sRNA-dependent regulation of the Salmonella ompD mRNA, the kinetics of an- nealing of three sRNAs, RybB, SdsR, and MicC, to the coding region of $o m p D$ mRNA were compared. Moreover, the truncated variants of these sRNAs were used to dissect the contributions of $\mathrm{Hfq}$ to the formation of each individual pair. Finally, the RNA structure probing was used to map the secondary structure of $o m p D$ mRNA and to monitor the conformational changes induced by the sRNA annealing.

\section{RESULTS}

The analysis of the sequence of $o m p D$ mRNA showed that its 5 -terminal region containing the binding sites of RybB, SdsR, and MicC sRNAs includes several sequence motifs that could serve as Hfq binding sites (Fig. 1). Among them are six $(\mathrm{ARN})_{2}$ repeats (named below as $A R N-1$ to $A R N-6$ ) located in the $5^{\prime}$-UTR and in the coding sequence. One of those motifs (termed ARN-3) is followed by a long AUrich region located mainly in the $5^{\prime}$-UTR (Fig. 1A). Both $A R N$ repeats and $A U$-rich sequences can serve as $\mathrm{Hfq}$ binding sites in mRNA molecules. For example, an (ARN) $)_{4}$ motif is important for $\mathrm{Hfq}$ binding and DsrA sRNA annealing to rpoS mRNA (Soper and Woodson 2008), an AU-rich sequence is involved in the regulation of chiP mRNA, while both ARN repeats and AU-rich regions serve in the regulation of $\operatorname{csg} D$ mRNA by different sRNAs (Schu et al. 2015). Hence, these data suggest that the ompD mRNA leader sequence could contain potential Hfq binding sites.

\section{Hfq binds tightly to the ompD mRNA}

To determine the affinity of Hfq to the ompD mRNA, a native gel mobility shift assay was used (Table 1; Fig. 2). In these experiments the 187-nt long 5 -terminal fragment of ompD mRNA (ompD-187) was used, which contains the binding sites of RybB, SdsR, and MicC sRNAs (Fig. 1). This ompD fragment was sufficient for the in vivo regulation of translation by MicC sRNA, which binds the deepest of them in the coding sequence (Pfeiffer et al. 2009). The data showed that $\mathrm{Hfq}$ formed three complexes with ompD-187 at a range of Hfq concentrations up to $5 \mathrm{nM}$ (Fig. 2A). The formation of higher order complexes with $\mathrm{Hfq}$ has also been previously observed for other RNAs, for example, for E. coli rpoS mRNA (Soper and Woodson 2008), RNA-IN mRNA (Ross et al. 2013), and DsrA sRNA (Lease and Woodson 2004). Hfq bound ompD mRNA leader very tightly with the equilibrium dissociation constant $\left(K_{\mathrm{d}}\right)$ value of $0.61 \pm 0.17 \mathrm{nM}$ for the tightest complex (per hexamer). This was similar to the $K_{\mathrm{d}}$ value of $0.24 \mathrm{nM}$ reported for the 160-nt long fragment of RNA-IN mRNA (Ross et al. 2013), and tighter than the $50 \mathrm{nM}$ value reported for the 301-nt long fragment of rpoS mRNA (Peng et al. 2014b) or the $30 \mathrm{nM}$ value reported for the 182-nt long fragment of $g l m S$ mRNA (Salim et al. 2012). These results demonstrated the direct binding of Hfq to ompD mRNA (Fig. 2), which was suggested by Hfq coimmunoprecipitation studies (Sittka et al. 2008) and by 
A

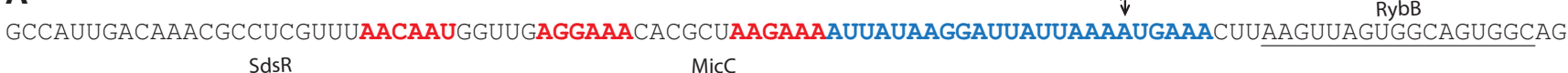
UGACUUCCCUGUUGGCAGCAGGCGUUGUAAAUGCAGCCGAGGUAUAUAACAAAGACGGCAAUAAACUGGAUCUGUACGGUAAAGUUCACG

B

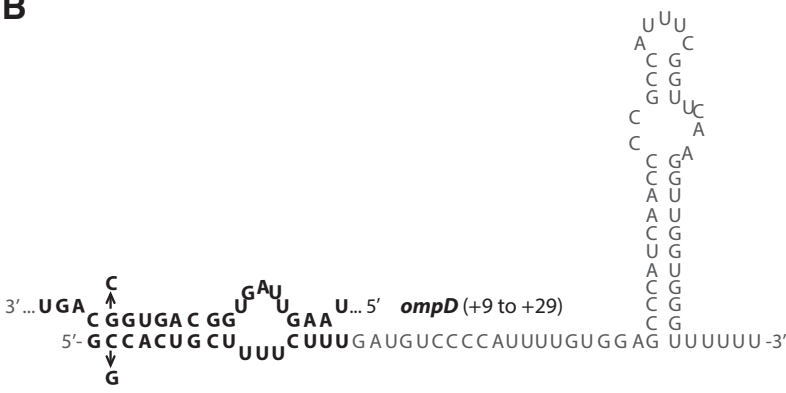

RybB

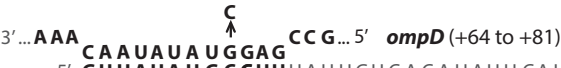

5'- GUUAUA G C CUUUAUUGUCACAUAUU CAUUUUGUC GUAUAAAUUGACA U UUUUU -3' MicC
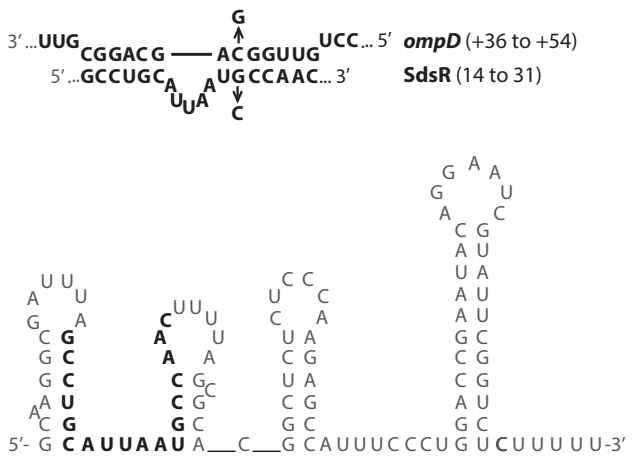

SdsR

FIGURE 1. The leader region of $o m p D$ mRNA and the secondary structures of sRNAs RybB, SdsR, and MicC. (A) The sequence of the $o m p D-187$ mRNA. The RybB, SdsR, and MicC sRNA binding sites are underlined. (ARN) $)_{2}$ motifs are marked with red font, and the AU-rich region with blue font. (B) Secondary structures of sRNAs RybB (Bouvier et al. 2008), MicC (Pfeiffer et al. 2009), and SdsR (Supplemental Fig. S1), with pairing to complementary ompD mRNA sequences shown on the structures of RybB and MicC, and above the structure of SdsR. The pairing region of SdsR is marked on its structure with bold font. The location of complementary regions within the ompD coding sequence is shown in brackets. The mutations used to confirm the sRNA-mRNA interactions (Supplemental Fig. S2) are shown at the structures of complementary regions.

Hfq binding to the binary complexes of ${ }^{32}$ P-labeled RybB with unlabeled $o m p D$ (Papenfort et al. 2006). Moreover, these results suggested a specific interaction of Hfq with ompD mRNA, because Hfq bound this mRNA with subnanomolar affinity (Table 1) comparable to those of other mRNAs, for which the functional role of Hfq binding was previously determined (Ross et al. 2013; Peng et al. 2014a).

To better understand how Hfq interacts with ompD mRNA, the binding of ompD-187 to wild-type (wt) Hfq and its variants with mutations in the distal (Y25D), proximal (K56A), or rim (R16A) surface of the ring was compared (Table 1). The $K_{\mathrm{d}}$ value for the tightest complex of ompD-187 with Hfq Y25D was threefold weaker than with wt Hff. At the same time, the $K_{\mathrm{d}}$ value for the tightest complex with $\mathrm{Hfq} \mathrm{K} 56 \mathrm{~A}$ was twofold weaker, and with Hfq R16A was not changed as compared to the
TABLE 1. Equilibrium binding of wt $\mathrm{Hfq}$ and its mutants to the ompD mRNA fragments and sRNA molecules

\begin{tabular}{lcccc}
\hline & \multicolumn{4}{c}{$K_{d}(\mathrm{nM})$} \\
\cline { 2 - 5 }${ }^{32}$ P-RNA & Hfq wt & Hfq Y25D & Hfq K56A & Hfq R16A \\
\hline RybB & $0.079 \pm 0.031^{\mathrm{a}}$ & $0.072 \pm 0.001^{\mathrm{a}}$ & $8.0 \pm 2.4^{\mathrm{a}}$ & $0.23 \pm 0.085^{\mathrm{a}}$ \\
SdsR & $0.052 \pm 0.009^{\mathrm{a}}$ & $0.097 \pm 0.013^{\mathrm{b}}$ & $3.1 \pm 0.25^{\mathrm{a}}$ & $0.028 \pm 0.005^{\mathrm{a}}$ \\
& & $0.76 \pm 0.015^{\mathrm{b}}$ & & \\
MicC & $0.078 \pm 0.024^{\mathrm{b}}$ & $0.056 \pm 0.014^{\mathrm{b}}$ & $4.1 \pm 1.5^{\mathrm{a}}$ & $0.044 \pm 0.006^{\mathrm{a}}$ \\
& $3.9 \pm 0.7^{\mathrm{b}}$ & $1.6 \pm 0.16^{\mathrm{b}}$ & & \\
ompD-187 & $0.61 \pm 0.17^{\mathrm{c}}$ & $1.6 \pm 0.27^{\mathrm{c}}$ & $1.1 \pm 0.21^{\mathrm{c}}$ & $0.46 \pm 0.09^{\mathrm{c}}$ \\
& $1.9 \pm 0.58^{\mathrm{c}}$ & $2.0 \pm 0.053^{\mathrm{c}}$ & $2.1 \pm 0.38^{\mathrm{c}}$ & $1.2 \pm 0.21^{\mathrm{c}}$ \\
ompD-187 ARN-3 & $0.93 \pm 0.24^{\mathrm{c}}$ & $3.4 \pm 0.4^{\mathrm{c}}$ & $1.6 \pm 0.47^{\mathrm{c}}$ & $3.1 \pm 0.11^{\mathrm{c}}$ \\
& $2.9 \pm 0.35^{\mathrm{c}}$ & $9.1 \pm 1.6^{\mathrm{c}}$ & $3.4 \pm 0.42^{\mathrm{c}}$ & $8.1 \pm 0.055^{\mathrm{c}}$ \\
ompD-187-SL2mut & $1.8 \pm 0.17^{\mathrm{c}}$ & $1.4 \pm 0.2^{\mathrm{c}}$ & $2.5 \pm 0.63^{\mathrm{c}}$ & $5.5 \pm 0.91^{\mathrm{c}}$ \\
& $3.4 \pm 1.1^{\mathrm{c}}$ & $7.6 \pm 1.9^{\mathrm{c}}$ & $11 \pm 0.61^{\mathrm{c}}$ & $21 \pm 2.8^{\mathrm{c}}$ \\
ompD-1-95 & $0.39 \pm 0.07^{\mathrm{a}}$ & n.m. & n.m. & n.m. \\
ompD-35-131 & $0.12 \pm 0.01^{\mathrm{a}}$ & n.m. & n.m. & n.m. \\
ompD-93-187 & $3.2 \pm 1.5^{\mathrm{a}}$ & n.m. & n.m. & n.m. \\
\hline
\end{tabular}

The numbers are averages of at least three independent experiments. (n.m.) Not measured. ${ }^{a}$ One complex with Hfq was formed; data were fit to the Michaelis-Menten binding isotherm.

${ }^{\mathrm{b}}$ Two complexes with Hfq were formed; data were fit to a partition function assuming two unequal independent binding sites.

'Three complexes with $\mathrm{Hfq}$ were formed; data were fit to a partition function assuming one specific and two equal nonspecific binding sites. 
A

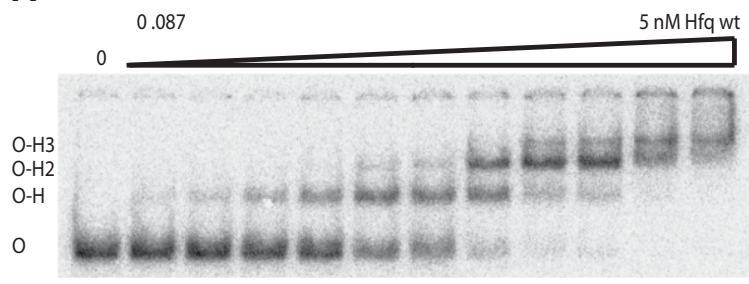

B

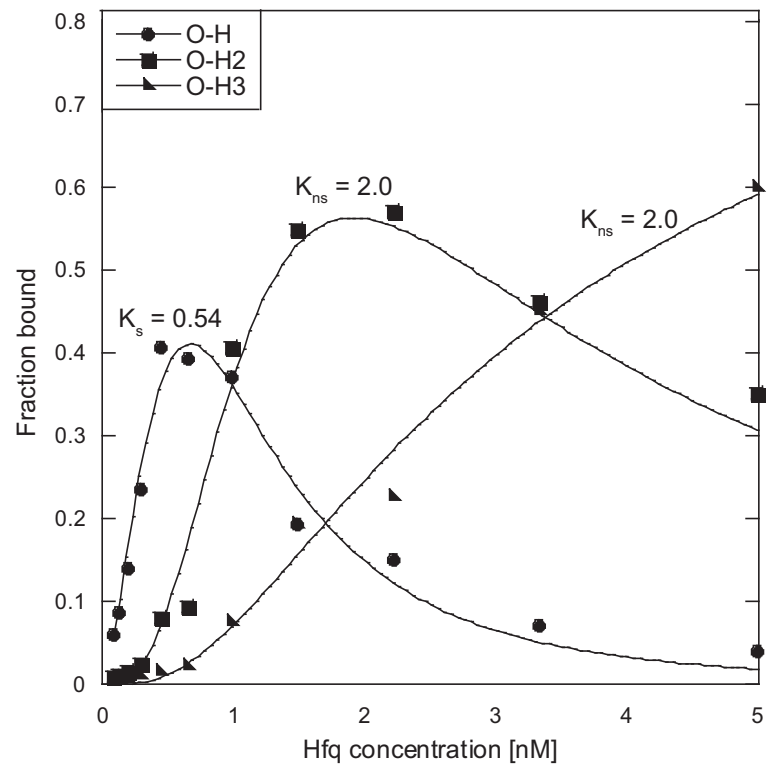

FIGURE 2. The equilibrium binding of Hfq to the ompD mRNA leader. (A) The analysis of Hfq binding to ${ }^{32} \mathrm{P}$-labeled ompD-187 using the native mobility shift assay. The ompD-187 complexes with Hfq are marked as $\mathrm{O}-\mathrm{H}, \mathrm{O}-\mathrm{H} 2$, and $\mathrm{O}-\mathrm{H} 3$. (B) The plot of ${ }^{32} \mathrm{P}-$ ompD-187 binding data from $A$ versus the concentration of Hfq. The data were fit to a partition function assuming one specific and two equal nonspecific binding sites. The equilibrium dissociation constant values obtained by fitting the data are shown on the plot, and the average values are presented in Table 1.

wild-type protein (Table 1). The modest effect of the Y25D mutation on the distal face indicated that other RNA binding sites on $\mathrm{Hfq}$ were involved, and partly compensated for the loss of the thermodynamic contribution of the distal surface contacts. The simultaneous use of several sites on Hfq was also observed for its binding to fhlA mRNA (Salim and Feig 2010), where both the distal and the proximal surfaces were used, and to rpoS mRNA (Peng et al. 2014a) and $\operatorname{csg} D$ mRNA (Schu et al. 2015), where both the distal and the rim surfaces were involved. These data suggest that the involvement of several RNA binding sites on the Hfq ring is a general feature of $\mathrm{Hfq}$ interactions with mRNA molecules.

The binding of Hfq to sRNAs RybB, SdsR, and MicC was mainly dependent on the proximal face contacts (Table 1). These sRNAs bound to wt Hfq with very tight affinities similar to those determined before for E. coli sRNAs (Olejniczak 2011). The Y25D mutation in the distal face of Hfq did not affect their binding, while the K56A mutation in the proximal face resulted in up to 100 -fold weaker binding. The R16A mutation on the rim of Hfq showed a small effect on the binding of RybB, but not of SdsR or MicC. The use of the proximal surface for binding to $\mathrm{Hfq}$ is characteristic of Class I sRNAs (Zhang et al. 2013; Schu et al. 2015), which are directed to this site by their $3^{\prime}$-terminal oligouridine sequences remaining from Rho-independent terminators (Sauer and Weichenrieder 2011). Consistently, the sRNAs studied here do not contain ARN repeats (Fig. 1B), which direct other sRNAs to the distal face of Hfq (Olejniczak 2011; Małecka et al. 2015; Schu et al. 2015). Overall, these data suggest that Hfq could use the proximal surface of its ring to recruit RybB, SdsR, and MicC sRNAs toward the ompD mRNA coding sequence.

\section{$\mathrm{Hfq}$ accelerates the annealing of RybB, SdsR, and MicC sRNAs to the ompD mRNA}

To elucidate the role of Hfq for the annealing of sRNAs to the coding sequence of $o m p D$ mRNA, the kinetics of association of RybB, SdsR, and MicC sRNAs to the $5^{\prime}{ }^{32} \mathrm{P}$-labeled ompD187 mRNA were analyzed (Fig. 3). The progress of annealing reactions was monitored using a native gel mobility shift assay in electrophoretic buffer containing $2 \mathrm{mM} \mathrm{Mg}^{2+}$ as previously described (Peng et al. 2014b). The rates of association $\left(k_{\text {obs }}\right)$ were measured at $1 \mathrm{nM}$ concentration of ${ }^{32} \mathrm{P}$-labeled ompD-187 and $25 \mathrm{nM}$ unlabeled sRNA in the presence or absence of $3 \mathrm{nM} \mathrm{Hfq}$. For each of the three studied sRNAs, the control reactions showed distinct differences in the electrophoretic mobility of free ompD-187, its binary complexes with Hfq or sRNA, and the ternary complex of ompD-187 with Hfq and sRNA.

The rate of RybB sRNA annealing to the $m p D$ mRNA leader was strongly accelerated by Hfq (Table 2; Fig. 3A,D). In the absence of $\mathrm{Hfq}$, the rate of RybB sRNA annealing to ${ }^{32} \mathrm{P}$-labeled ompD-187 was $0.032 \mathrm{~min}^{-1}$. However, when $3 \mathrm{nM}$ Hfq was present, a ternary complex was formed rapidly with a rate that was more than 250-fold faster (Table 2; Fig. 3A, D). This effect was comparable to that for DsrA annealing to a 301-nt fragment of rpoS mRNA, where Hfq induced 60fold faster association (Peng et al. 2014b). To test whether base-pairing between $\mathrm{RybB}$ and $o m p D-187$ was required to form the ternary complex with $\mathrm{Hfq}$, the effect of mutations in the complementary regions of RybB (mutation $\mathrm{C} 2 \mathrm{G}$ ) and ompD-187 (mutation G94C) was studied (Fig. 1B). These mutations were detrimental for translation regulation in vivo (Bouvier et al. 2008). Indeed, the RybB C2G mutant failed to form a complex with ompD-187 mRNA (Supplemental Fig. S2A), and the annealing of RybB to the ompD-187 G94C mutant was weakened in comparison to the natural ompD-187 sequence (Supplemental Fig. S2B). However, the annealing of RybB C2G mutant to ompD-187 G94C mutant was restored to the wild-type level (Supplemental Fig. S2C). This confirms that in the ternary complex RybB is directly 
A

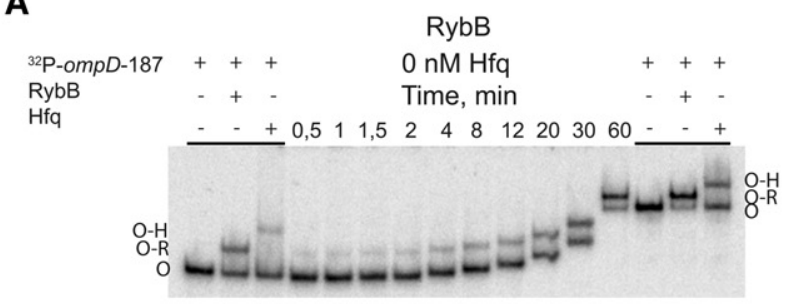

RybB

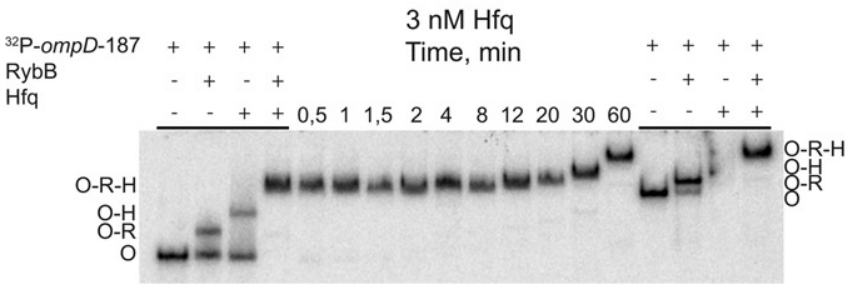

C
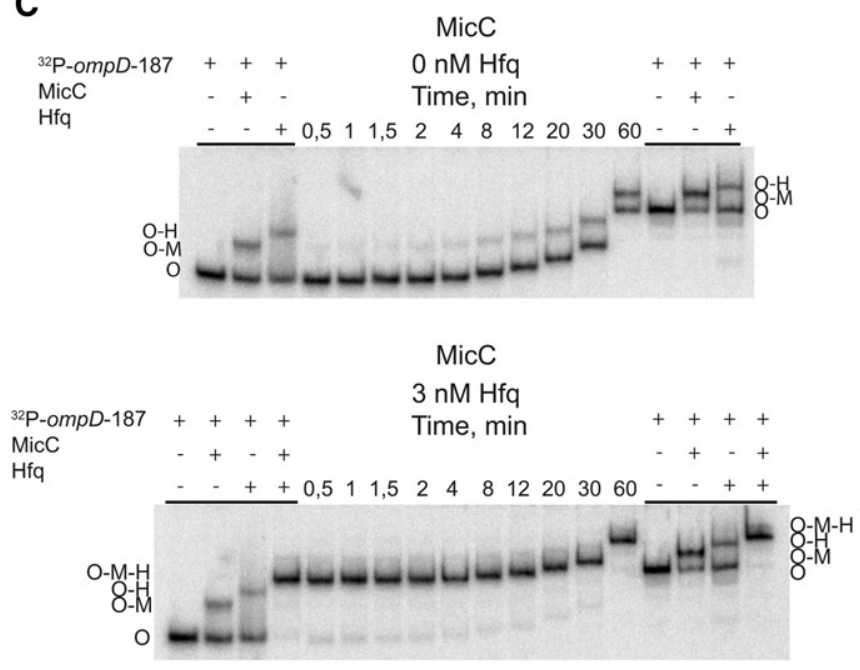

B

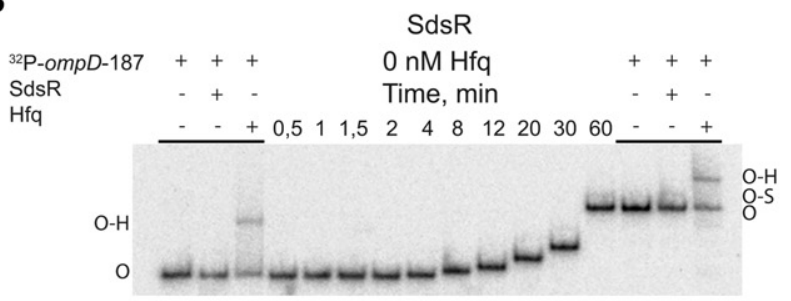

SdsR

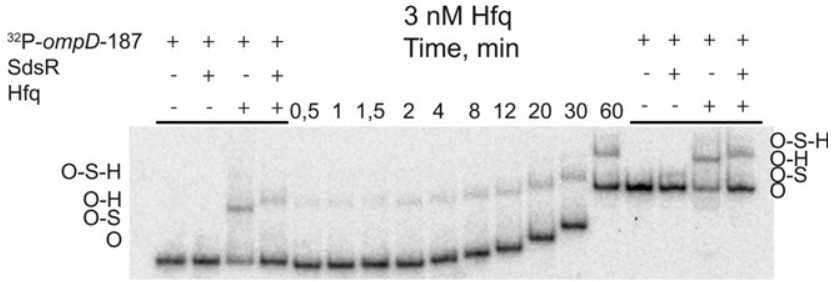

D

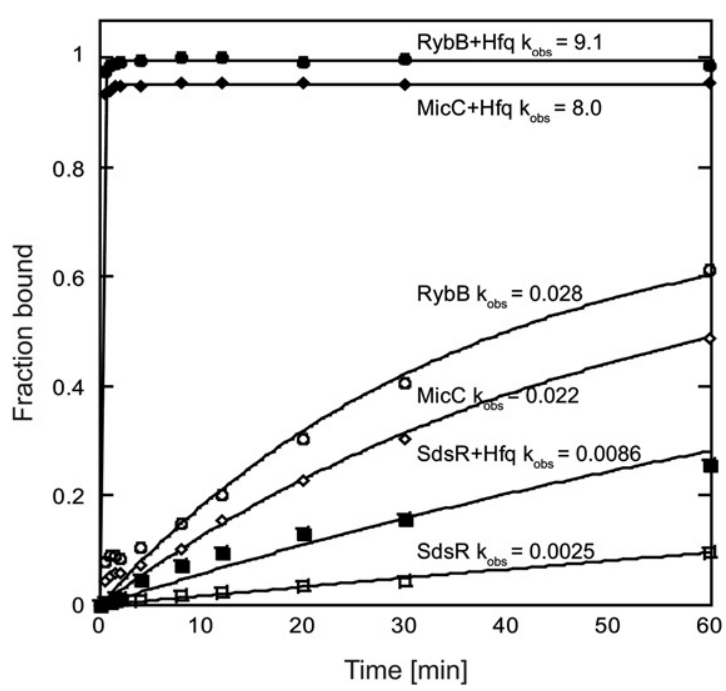

FIGURE 3. Hfq accelerates the association of RybB, SdsR, and MicC sRNAs to ompD mRNA. The kinetics of annealing of ${ }^{32} \mathrm{P}-\mathrm{labeled}$ ompD-187 ( $1 \mathrm{nM}$ concentration) to $(A) \mathrm{RybB},(B) \mathrm{SdsR}$, or $(C)$ MicC sRNA at $25 \mathrm{nM}$ concentration, in the absence or presence of $3 \mathrm{nM}$ Hfq. Free ompD-187 is marked as $\mathrm{O}$, ompD-187-Hfq complex as $\mathrm{O}-\mathrm{H}$, ompD-187-RybB complex as O-R, ompD-187-RybB-Hfq ternary complex as O-R-H, ompD-187-SdsR complex as O-S, ompD-187-SdsR-Hfq ternary complex as O-S-H, ompD-187-MicC complex as O-M, and ompD-187-MicC-Hfq ternary complex as O-M-H. The control reactions, in which ${ }^{32} \mathrm{P}-o m p D-187$ alone was bound to Hfa, were supplemented with $2 \mathrm{nM}$ cold ompD-187 mRNA to obtain the total concentration of RNA equal to that of Hfq. $(D)$ The data from $A, B$, and $C$ were plotted versus time, and the fitting of data provided $k_{\mathrm{obs}}$ values, which are presented on the plot. The average $k_{\mathrm{obs}}$ values are shown in Table 2 .

paired to ompD-187, as opposed to binding independently to different sites on Hfq.

The annealing of SdsR sRNA to the $\operatorname{mom}_{p} D \mathrm{mRNA}$ leader in the presence of Hfq proceeded much more slowly than that of RybB (Table 2; Fig. 3B,D). In the absence of Hfa, SdsR sRNA annealed to $o m p D-187$ with a rate of $0.0029 \mathrm{~min}^{-1}$. In the presence of Hfq, a ternary complex was formed with only a threefold faster rate (Table 2). The mutations in complementary positions in SdsR (the G26C mutation) and in ompD-187 (the $\mathrm{C} 113 \mathrm{G}$ mutation) were detrimental for annealing when tested individually (Supplemental Fig. S2D,E), in agreement with their effect on translation regulation (Frohlich et al. 2012). Consistently, the annealing was restored to the wild- type level when molecules with compensatory mutations were studied (Supplemental Fig. S2F).

Hfq also had a strong influence on the rates of MicC sRNA annealing to $o m p D$ (Table 2; Fig. 3C,D). In the absence of Hfq, the rate of MicC annealing to ${ }^{32} \mathrm{P}$-labeled $o m p D-187$ was $0.025 \mathrm{~min}^{-1}$. In the presence of $\mathrm{Hfq}$, the annealing rate was more than 250-fold faster, which was comparable to the effect observed for RybB annealing (Table 2). The mutations in complementary positions in $\mathrm{MicC}$ (the $\mathrm{C} 9 \mathrm{G}$ mutation) and in ompD-187 (the G139C mutation) were detrimental for annealing when tested individually (Supplemental Fig. S2G,H), in agreement with their effect on $\operatorname{ompD}$ regulation in vivo (Pfeiffer et al. 2009). The MicC sRNA annealing to mRNA 
TABLE 2. The rates of annealing of full-length and truncated sRNA molecules to ompD-187 and its fragments ${ }^{\mathrm{a}}$

\begin{tabular}{|c|c|c|}
\hline \multirow[b]{2}{*}{ Interacting RNAs } & \multicolumn{2}{|c|}{$k_{\text {obs }}\left(\min ^{-1}\right)$} \\
\hline & $-\mathrm{Hfq}$ & $+\mathrm{Hfq}$ \\
\hline${ }^{32} \mathrm{P}-o m p D-187-$ RybB & $0.032 \pm 0.005$ & $8.8 \pm 0.7$ \\
\hline${ }^{32} \mathrm{P}-o m p D-21 \mathrm{R}$-RybB & $\begin{array}{l}3.3 \pm 0.65^{\mathrm{b}} \\
0.2 \pm 0.033^{\mathrm{b}}\end{array}$ & $3.0 \pm 1.2$ \\
\hline${ }^{32}$ P-RybB-16—ompD-187 & $\begin{array}{c}1.9 \pm 0.17^{\mathrm{b}} \\
0.14 \pm 0.047^{\mathrm{b}}\end{array}$ & $2.2 \pm 0.51$ \\
\hline${ }^{32} \mathrm{P}-\mathrm{ompD}$-187-SdsR & $0.0029 \pm 0.0008$ & $0.01 \pm 0.002$ \\
\hline${ }^{32} \mathrm{P}-\mathrm{ompD}-19 \mathrm{~S}$-SdsR & $0.005 \pm 0.0002$ & $0.0067 \pm 0.0006$ \\
\hline${ }^{32}$ P-SdsR-18-ompD-187 & $0.015 \pm 0.0023$ & $0.015 \pm 0.0011$ \\
\hline${ }^{32} \mathrm{P}-\mathrm{ompD}-187-\mathrm{MicC}$ & $0.025 \pm 0.0037$ & $6.9 \pm 0.79$ \\
\hline${ }^{32} \mathrm{P}-\mathrm{ompD}-18 \mathrm{M}-\mathrm{MicC}$ & $\begin{aligned} 3.3 & \pm 1.3 \\
0.072 & \pm 0.0049\end{aligned}$ & $\begin{aligned} 2.0 & \pm 0.065^{c} \\
0.17 & \pm 0.06^{c}\end{aligned}$ \\
\hline${ }^{32} \mathrm{P}-\mathrm{MicC}-12-\mathrm{ompD}-187$ & $3.3 \pm 1.6$ & $3.8 \pm 1.1$ \\
\hline
\end{tabular}

The numbers are averages of at least three independent experiments.

${ }^{\mathrm{a}}$ The concentration of ${ }^{32} \mathrm{P}$-labeled RNA was $1 \mathrm{nM}$, Hfq $3 \mathrm{nM}$, and unlabeled RNA $25 \mathrm{nM}$.

${ }^{\mathrm{b}}$ The data were fit using a biphasic exponential equation, with $60 \%$ in the fast phase.

${ }^{\mathrm{c}}$ The data were fit using a biphasic exponential equation, with $40 \%$ in the fast phase.

was restored, when molecules with compensatory mutations were used (Supplemental Fig. S2I). Overall, these data showed that the annealing rates of all three sRNAs were increased by $\mathrm{Hfq}$, although the effects were ranging from as little as threefold for SdsR to more than 200-fold for RybB and MicC.

To elucidate the involvement of Hfq binding sites, the annealing of RybB, SdsR, and MicC sRNAs to ompD-187 was measured in the presence of $\mathrm{Hfq}$ variants with mutations in the sites of RNA binding (Table 3). The data showed that both the mutation Y25D in the distal face and the mutation $\mathrm{K} 56 \mathrm{~A}$ in the proximal face resulted in more than 100-fold slower annealing of RybB to ompD-187, while the rim mutation had a small effect. The annealing of MicC was also affected by both the distal and the proximal face mutations, which had about a 25 -fold effect, while the rim mutation had a fivefold effect (Table 3). As the annealing of SdsR in the presence of wt Hfq was already slow, the Hfq mutations in the proxi- mal and distal face had only about a twofold effect, and the rim mutation did not affect the annealing. Because the equilibrium binding data showed absolute preference of sRNAs $\mathrm{RybB}, \mathrm{SdsR}$, and MicC to bind to the proximal face of $\mathrm{Hfq}$ (Table 1), this suggested that ompD mRNA binds to the distal face of Hfq, in agreement with the detrimental effect of the Y25D mutation on the annealing (Table 3).

\section{Hfq differently contributes to the annealing of sRNAs RybB, SdsR, and MicC to ompD mRNA}

The role of Hfq in the annealing of each sRNA to ompD mRNA was further dissected using minimal sRNA molecules and complementary ompD mRNA fragments (Table 2; Fig. 1B; Supplemental Fig. S3). The study of the annealing of truncated variants of interacting RNAs should allow us to evaluate the contributions of their structures to the energetic barrier preventing their annealing in the absence of Hfq. A similar approach has been used previously to reveal that the essential role of $\mathrm{Hfq}$ in DsrA sRNA annealing to rpoS mRNA was to rearrange the rpoS mRNA structure (Soper et al. 2011). The minimal sRNA molecules consisting of the sequence that is complementary to ompD (shown in bold in Fig. 1B) were RybB-16, which was effective in repression of ompN mRNA translation (Bouvier et al. 2008), SdsR-18, and MicC-12. The minimal fragments of $o m p D$ mRNA corresponding to the binding sites of these sRNAs were ompD$21 \mathrm{R}$, ompD-19S, and ompD-18M, respectively. None of the six short RNAs bound Hfq at the concentrations used in the annealing assays (Supplemental Fig. S3).

The data suggested that the structures of both RybB sRNA and $o m p D$ mRNA contributed to the energetic barrier preventing their efficient annealing (Table 2; Supplemental Fig. S3). In the absence of Hfa, both the truncated RybB16 fragment and the truncated ompD-21R fragment bound to $o m p D-187$ and RybB, respectively, much faster than fulllength RybB to ompD-187 (Table 2; Supplemental Fig. S3A, $\mathrm{B}, \mathrm{G}, \mathrm{H})$. In the presence of $\mathrm{Hfq}$, both truncated fragments bound to the full-length molecules faster than in its absence, with rates that were comparable to that of full-length RybB annealing to ompD-187 (Table 2). These results suggested

TABLE 3. The rates of sRNA annealing to $o m p D$ mRNA in the presence of Hfq mutants

\begin{tabular}{lccccc}
\hline & \multicolumn{4}{c}{$k_{\text {obs }}\left(\mathrm{min}^{-1}\right)$} \\
\cline { 2 - 6 } Interacting RNAs & - Hfq & +Hfq wt & +Hfq Y25D & +Hfq K56A & + +Hfq R16A \\
\hline${ }^{32} \mathrm{P}-$ ompD-187-RybB & $0.032 \pm 0.005^{\mathrm{a}}$ & $8.8 \pm 0.7^{\mathrm{a}}$ & $0.049 \pm 0.0088$ & $0.045 \pm 0.0039$ & $2.9 \pm 0.3$ \\
${ }^{32} \mathrm{P}-$-ompD-187 SL2mut-RybB & $0.011 \pm 0.0018$ & $0.036 \pm 0.007$ & $0.0097 \pm 0.0024$ & $0.011 \pm 0.0018$ & $0.058 \pm 0.0078$ \\
${ }^{32} \mathrm{P}-$ ompD-187-SdsR & $0.0029 \pm 0.0008^{\mathrm{a}}$ & $0.01 \pm 0.0021^{\mathrm{a}}$ & $0.0046 \pm 0.0002$ & $0.0062 \pm 0.0013$ & $0.017 \pm 0.0039$ \\
${ }^{32} \mathrm{P}-$-ompD-187 SL2mut-SdsR & $<0.001$ & $<0.001$ & $<0.001$ & $<0.001$ & $<0.001$ \\
${ }^{32} \mathrm{P}-$ ompD-187-MicC & $0.025 \pm 0.0037^{\mathrm{a}}$ & $6.9 \pm 0.79^{\mathrm{a}}$ & $0.26 \pm 0.039$ & $0.28 \pm 0.005$ & $1.2 \pm 0.02$ \\
${ }^{32} \mathrm{P}-$-ompD-187 SL2mut-MicC & $0.0088 \pm 0.002$ & $0.032 \pm 0.0095$ & $0.012 \pm 0.0005$ & $0.0082 \pm 0.0011$ & $0.02 \pm 0.0039$ \\
\hline
\end{tabular}

${ }^{a}$ Values from Table 2. 
that the role of Hfq in the annealing of RybB to ompD-187 was to rearrange the structures of both interacting RNAs.

The contribution of Hfq to the annealing of SdsR sRNA to ompD-187 depended on the structures of both interacting molecules, but the effects of the RNA truncation were relatively modest (Table 2; Supplemental Fig. S3C,D,G,H). In the absence of Hfa, the rates of SdsR-18 annealing to ompD-187 were fivefold faster, and those of ompD-19S annealing to SdsR were twofold faster than for the annealing of SdsR to ompD-187. This suggested that the role of Hfq in the full-length SdsR annealing to $o m p D-187$ was to rearrange the structures of both interacting RNAs. However, Hfq did not further increase the rates of annealing of these RNA pairs.

In contrast, the structure of MicC sRNA was the main barrier to its annealing to ompD-187 (Table 2; Supplemental Fig. $\mathrm{S} 3 \mathrm{E}, \mathrm{F}, \mathrm{G}, \mathrm{H})$. Both in the absence and presence of Hfq the rates of annealing of minimal MicC-12 to ompD-187 were similar to the rates of full-length MicC annealing to $o m p D$ 187 in the presence of Hfq. This suggested that in the context of the structure of ompD-187, the MicC binding site was easily accessible for MicC-12. In contrast, both in the absence and presence of $\mathrm{Hfq}$ the truncated $o m p D-18 \mathrm{M}$ bound to fulllength MicC much slower than ompD-187 did. ompD-18M had biphasic rates of annealing to $\mathrm{MicC}$ with a faster rate responsible for $40 \%$ of the binding (Table 2; Supplemental Fig. S3). Overall, these data suggested that the structure of MicC could be an important barrier to its annealing to ompD mRNA, and thus would necessitate the use of $\mathrm{Hfq}$ for unfolding. Alternatively, a role of Hfq could be to optimally position the pairing sequence of the full-length $\mathrm{MicC}$ toward its binding site in $o m p D$ mRNA. Regardless of the detailed explanation, these data suggested that the contribution of Hfq to MicC sRNA annealing was mostly determined by the structure of $\mathrm{MicC}$, and not by the structure of ompD mRNA.

\section{The ompD mRNA leader sequence folds into five stem-loops with RybB, SdsR, and MicC binding sites located in different structural contexts}

To elucidate how the structural context of sRNA binding sites could affect their annealing, the in vitro structure probing of the ompD-187 mRNA was performed using structure-specific ribonucleases (Fig. 4). Nuclease S1 and RNase T2 were used to identify structurally dynamic regions, while the location of the double-stranded regions was inferred from the comparison of RNase T1 induced cleavages in denaturing and native conditions, and from degradation induced by RNase III. The 3'-terminal 10-nt sequence of ompD-187 was predicted to be single-stranded, because the $3^{\prime}$-truncated 10-nt shorter derivative of ompD leader had the same pattern of cleavages as ompD-187 (data not shown). The probing data were used as constraints to predict the ompD mRNA structure using RNAstructure software (Reuter and Mathews
2010). The data showed that the $5^{\prime}$-terminal region of ompD mRNA was organized into five stem-loop structures, named below as SL1 to SL5 (Fig. 4B). The cleavage patterns of three shorter mRNA fragments containing the sequences corresponding to stem-loops SL1 and SL2 (ompD-1-95), SL2 and SL3 (ompD-35-131), or SL3, SL4, and SL5 (ompD93-187), were the same as the patterns of the corresponding regions in the 187-nt-long ompD mRNA leader (data not shown), which confirmed that the sequences involved in the predicted five stem-loop structures indeed formed separate structure modules.

The probing data showed that each of the three sRNA binding sites was located in a different structural context in ompD mRNA (Fig. 4). The binding site of RybB sRNA was located in a structurally dynamic SL2 motif, the binding site of SdsR in a stable apical region of SL3, and the binding site of MicC in an extended loop of SL4 (Fig. 4B). The 5'-terminal sequence and the apical loop of SL2, including the ARN-3 motif and the AU-rich region, were susceptible to cleavage by single-strand specific nucleases, which suggested that this region was quite dynamic. The RybB binding site was adjacent to the ARN-3 motif and the AU-rich region because it was located in the complementary strand of SL2. On the other hand, the binding site of SdsR sRNA, and the overlapping ARN-4 motif, were located in the apical portion of the SL3 motif (Fig. 4B). This element of ompD mRNA secondary structure was likely quite stable, because there were few cleavages with any used nucleases in this region, except for the apical triloop of SL3 (Fig. 4A).

The unique feature of the MicC sRNA binding site was its location in the extended purine-rich 16-nt apical loop of SL4, the stem of which was stabilized by four GC pairs (Fig. 4). The loop of SL4 was defined by a series of strong cleavages by RNase T2 and nuclease S1 at residues 140-144. The absence of cleavages at $145-150$ could indicate the presence of noncanonical interactions within the loop. The singlestranded character of this region was further supported by degradations induced in native conditions by RNase $\mathrm{T} 1$ at G138 and G139 at the $5^{\prime}$ end of this loop, and at residue G151 at its $3^{\prime}$ end. The presence of the stable stem of SL4 was supported by the decreased $\mathrm{T} 1$ cleavage in native conditions at residue G133. The ARN-5 motif, which partly overlaps the MicC binding site, was located in the loop of SL4, and the nearby ARN-6 motif was located in the single-stranded region between SL4 and SL5.

\section{The annealing of sRNAs RybB and MicC induces rearrangements in the structure of the $o m p D$ mRNA leader}

The annealing of RybB sRNA to $o m p D-187$ induced extensive changes in the cleavage pattern of stem-loops SL1 and SL2, and the intervening sequence (Fig. 5A,C). While the degradation of apical portions of SL1 and SL2 increased with the concentration of RybB, the linker between SL1 and SL2 together 

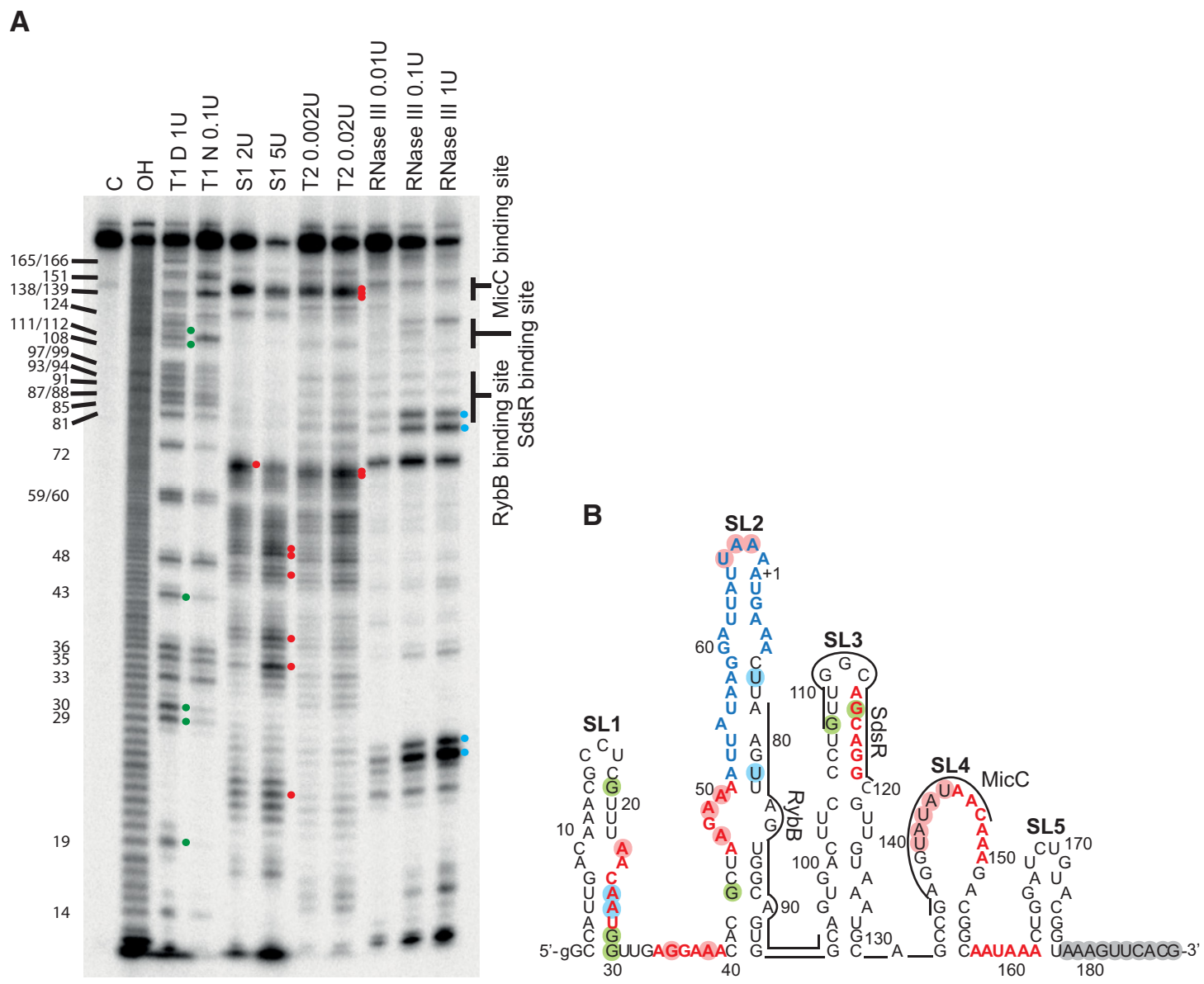

FIGURE 4. The binding sites of RybB, SdsR, and MicC sRNAs have different structural contexts within the ompD mRNA leader. (A) The structure probing of $5^{\prime}-{ }^{32} \mathrm{P}$-labeled ompD-187 using RNases indicated above the lanes. The untreated ompD-187 sample was resolved in lane C, formamide ladder in lane $\mathrm{OH}$, and the products of reactions with RNase T1 in denaturing or native conditions in lanes marked T1 D and T1 N, respectively. The positions of G-specific cleavages by RNase T1 are indicated on the left side of the gel. $(B)$ The secondary structure model of ompD-187 proposed by the RNAstructure program with hard constraints (circled positions) obtained from structure probing experiments in $A$. Residues constrained as single-stranded are indicated with red circles, while double-stranded are indicated with blue circles (RNase III) or green circles (RNase T1 in native conditions). Gray circles indicate nucleotides constrained as single-stranded based on experiments comparing the cleavage patterns of ompD-187 and $3^{\prime}$-end-truncated ompD-177 (data not shown). The AU-rich region is marked in blue, and (ARN) ${ }_{2}$ motifs in red. The binding sites of RybB, SdsR, and MicC sRNAs are indicated by lines.

with the adjacent regions became more protected (Fig. 5A,C). The increased cleavages at positions $79-81$ and $83-84$ (Fig. $5 \mathrm{~A}, \mathrm{C})$ marked the $5^{\prime}$ edge of the RybB binding site in mRNA. It was previously reported that the $5^{\prime}$ terminal sequence of RybB was complementary to two partly overlapping duplicated sequences in ompD mRNA, with the downstream sequence considered as the more likely binding site (Balbontin et al. 2010; Papenfort et al. 2010). The increased cleavages observed here are consistent with RybB binding to the downstream binding site, which confirms that conclusion. In contrast to the extensive conformational rearrangements induced by RybB, the structure-probing pattern of ompD-187 was not changed in the presence of SdsR sRNA (Supplemental Fig. S4). The lack of SdsR-dependent changes in the cleavage pattern suggests that the binding of SdsR protects this region from cleavage or that bound SdsR causes only limited unfolding of $o m p D$ mRNA structure.
The binding of MicC sRNA induced specific local changes in the probing pattern of the $3^{\prime}$ part of the SL4 loop and the adjacent stem (Fig. 5B,D). The nuclease S1 probing of the ompD mRNA fragment including the nucleotides from 93 to 187 (ompD-93-187) showed that the binding of MicC sRNA protected the apical loop nucleotides 137 and 140144 in SL4, which are within the MicC binding site. Interestingly, it also stimulated the cleavage of residues 149, 150, 152, and 153 (Fig. 5B), which are located immediately $3^{\prime}$ of the MicC binding site. The cytosine 153 is involved in the top base pair of the SL4 stem, which would be unfolded by MicC pairing to the sequence including complementary G136. In contrast, the adenosines 149, 150, and 152 are part of the sequence of five purines in the SL4 loop, which are not predicted to form canonical Watson-Crick interactions. However, large loops are often stabilized by noncanonical bonding patterns (Halder and Bhattacharyya 2013), and a 
A

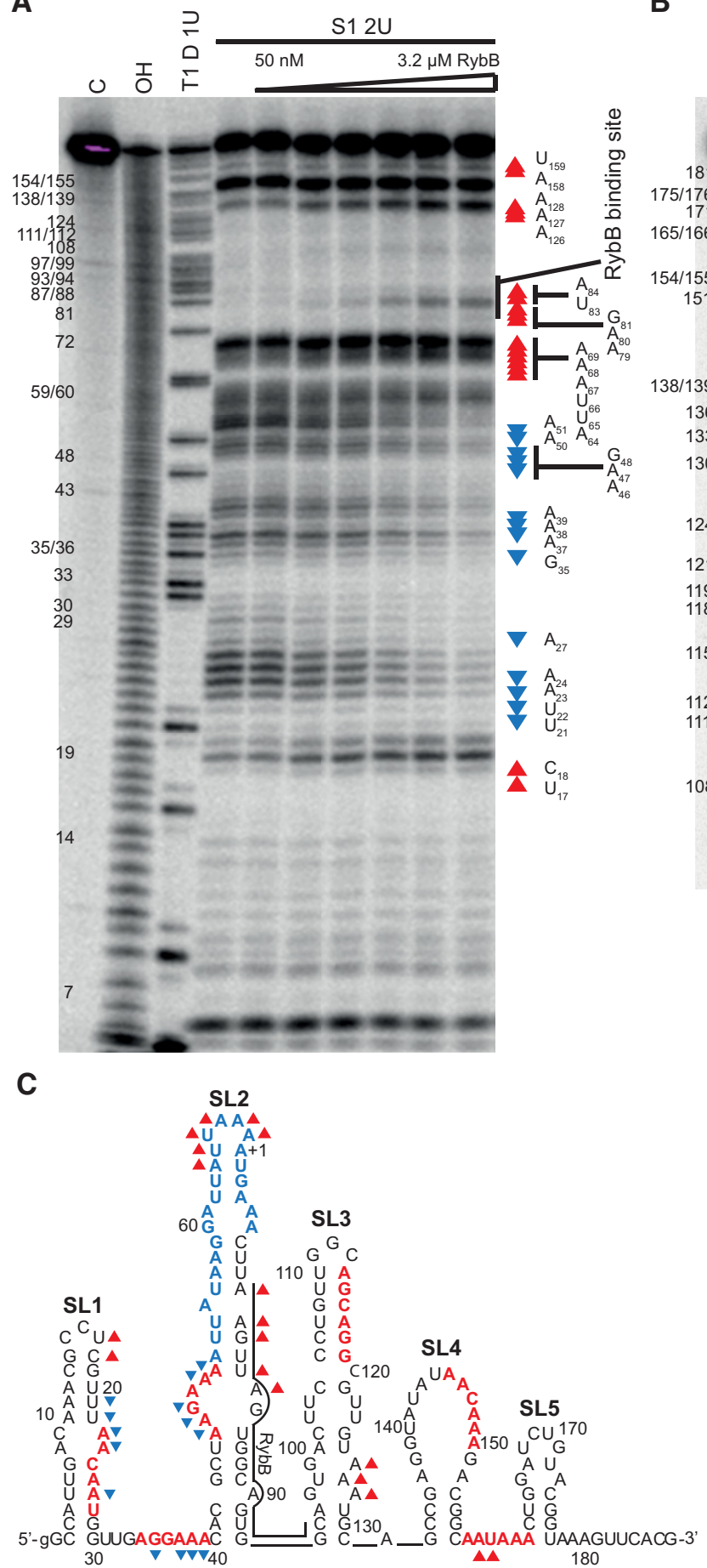

B

0 ○ $匚 \stackrel{20 \mathrm{nM} \quad 0.8 \mu \mathrm{MMicC}}{\longrightarrow}$

D

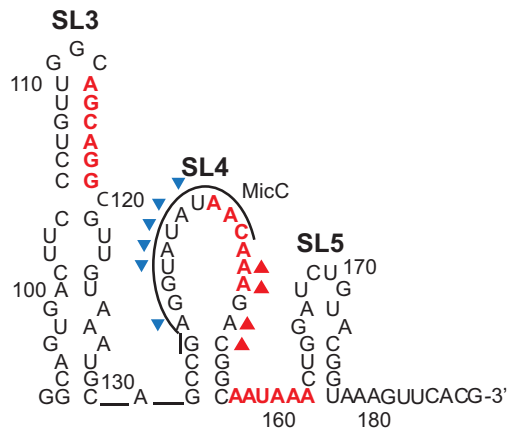

FIGURE 5. The binding of RybB and MicC sRNAs induces local conformational changes in the ompD mRNA leader. $(A)$ The structure probing of $5^{\prime}-{ }^{32} \mathrm{P}$-labeled $o m p D-187$ with nuclease S1 at increasing concentrations of RybB sRNA. (B) The structure probing of $5^{\prime}-{ }^{32} \mathrm{P}$-labeled ompD-93-187 with nuclease $\mathrm{S} 1$ at increasing concentrations of MicC sRNA. The untreated samples were resolved in lanes marked $\mathrm{C}$, formamide ladders in lanes $\mathrm{OH}$, and reactions with RNase T1 in denaturing conditions in lanes marked T1 D. The positions of G-specific cleavages by RNase T1 are indicated on the left sides of the gels. The changes in nucleotide susceptibility to cleavage upon RybB or MicC binding are shown on the secondary structure of ompD-187 in $C$, and ompD-93-187 in $D$, respectively. The nucleotide positions, which showed higher susceptibility to degradation by nuclease $S 1$ in the presence of respective sRNA, are marked by red triangles, and those which showed lower susceptibility are marked by blue reverse triangles.

continuous purine sequence is also expected to be stabilized by stacking interactions. The disruption of these intra-loop interactions by MicC binding would explain the increased susceptibility of this region to degradation by nuclease S1. Interestingly, the adenosine 152 (position 83 of the coding sequence) was also reported as a site of increased RNase E 
cleavage of ompD mRNA upon MicC binding in vivo (Pfeiffer et al. 2009). Hence, the data suggest that the annealing of MicC increases the conformational dynamics of the mRNA region $3^{\prime}$ adjacent to its binding site, in agreement with the increased susceptibility of this region to RNase E cleavage upon MicC binding in vivo.

\section{The $\mathrm{Hfq}$ binding site in the $5^{\prime}$-untranslated region of ompD mRNA is important for sRNA annealing to the coding sequence}

To identify the location of Hfq binding sites in ompD mRNA, a boundary binding assay was used (Fig. 6; Supplemental Fig. S5). This assay took advantage of the fact that Hfq remains tightly bound to its RNA ligands even in the presence of $8 \mathrm{M}$ urea (Brescia et al. 2003). After $5^{\prime}{ }^{32}$ P-labeled ompD187 was partly degraded with RNase T1 (Fig. 6) or nuclease S1 (Supplemental Fig. S5A), the reaction products were incubated with Hfq. This was followed by separation of

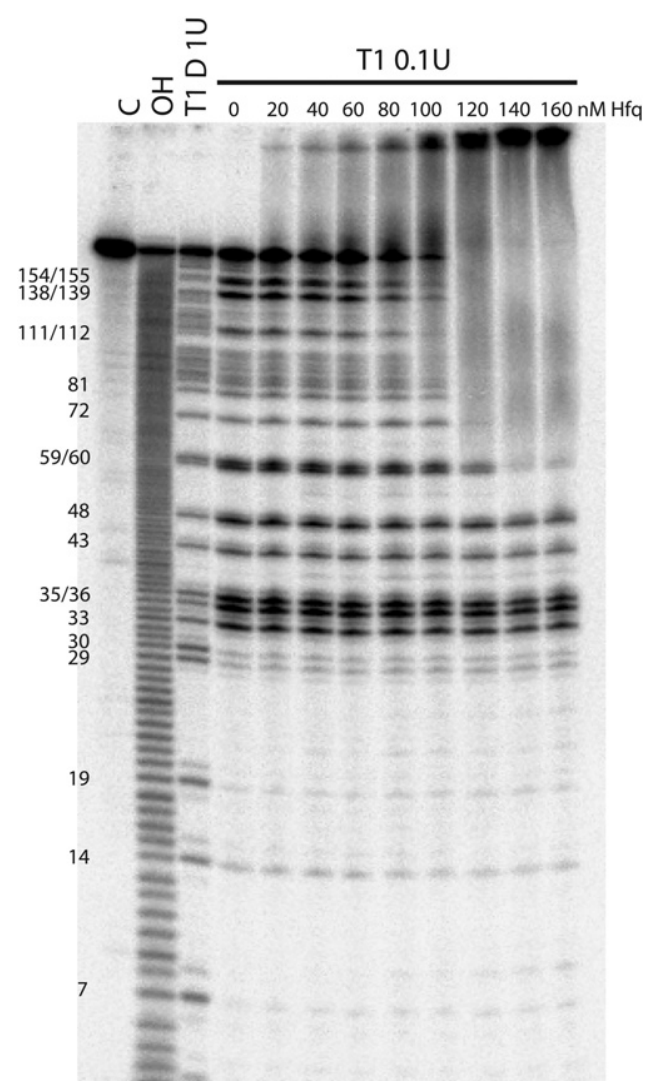

FIGURE 6. The boundary experiments with $5^{\prime}-{ }^{32} \mathrm{P}$-labeled $o m p D-187$ mRNA suggest that it contains a strong Hfq binding site in the $5^{\prime}$-untranslated region. The denaturing gel analysis of the binding of Hfq to the 5'-labeled $\operatorname{ompD}$-187 degradation fragments obtained by partial digestion with RNase T1. The untreated ompD-187 sample was resolved in the lane marked $\mathrm{C}$, the formamide ladder in lane $\mathrm{OH}$, and reaction with RNase T1 in denaturing conditions in lane T1 D. The positions of G-specific cleavages by RNase T1 are indicated on the left side of the gel.
Hfq-bound from unbound RNA fragments by denaturing gel electrophoresis (Fig. 6; Supplemental Fig. S5). At Hfq concentrations above $100 \mathrm{nM}$, only the $5^{\prime}$-terminal RNA fragments of at least $60 \mathrm{nt}$ in length were shifted by Hfq on a denaturing gel. This suggested the presence of a strong Hfq binding site in the $3^{\prime}$ part of this 60-nt long region, which coincides with the location of the sequence composed of ARN-3 motif followed by the AU-rich sequence. When the boundary experiment was performed using the $3^{\prime}$-end labeled ompD-187, the minimal fragments bound included the region from about position 110-187, which contained motifs ARN-5 and ARN-6 (Supplemental Fig. S5B,C). Overall, these data suggested that ompD-187 contains at least two Hfq binding sites, one of which is located in the untranslated region and the other in the coding sequence of this mRNA.

To investigate the relative strengths of these two potential Hfq binding sites, the affinities of $\mathrm{Hfq}$ for three overlapping fragments of ompD mRNA were determined (Table 1). The ompD-1-95 fragment contained regions SL1 and SL2, ompD-35-131 contained SL2 and SL3, and ompD-93-187 contained SL3, SL4, and SL5 (Fig. 4B). The data showed that the Hfq affinity for ompD-1-95 was similar to that for ompD-187, while the affinity for ompD-35-131 was fourfold tighter. Both of these constructs contained SL2, which included the ARN-3 motif followed by the AU-rich region. In contrast, the Hfq binding affinity of ompD-93-187, which contained motifs ARN-5 and ARN-6, was sixfold weaker than that of ompD-187. These data are consistent with the presence of an important Hfq binding site in SL2, which is present in both ompD-1-95 and ompD-35-187 mRNA fragments, but not in ompD-93-187.

To test whether ARN sequence repeats present at several regions of $o m p D$ mRNA participate in Hfq binding and sRNA annealing, the effect of point mutations in the $(\mathrm{ARN})_{2}$ motifs was analyzed (Supplemental Fig. S6; Supplemental Tables S1, S2). The secondary structure of ompD-187 mutants with substitutions in the repeats ARN-1, ARN-2, ARN-3, ARN-5, and ARN-6 was conserved, as predicted by RNAstructure software (Supplemental Fig. S6). The ARN-4 motif was not mutated, because it is located in a stable secondary structure region. The equilibrium binding data showed that none of the mutations affected the stability of the tightest complex of ompD-187 with Hfq (Supplemental Table S1). Only a modest, less than threefold decrease in the stability of the weaker complexes with Hfq was observed when both ARN-5 and ARN-6 were mutated. It is consistent with the observation that mutations in $(\mathrm{AAN})_{4}$ motif in rpoS mRNA had a detrimental effect on the formation of higher order complexes with Hfq (Peng et al. 2014b). When the rates of RybB annealing to ompD-187 mutants were measured, all of the mutants had a modest, less than threefold, detrimental effect on the annealing (Supplemental Table S2). The rates of MicC annealing to ompD-187 with ARN-1, ARN-2, or ARN3 mutations were similarly affected, while the mutations of 
ARN-5 and ARN-6 had much larger effects. However, because these mutations were adjacent to the MicC binding site, they could have affected its annealing directly and not via their influence on the Hfq binding. Moreover, it was previously proposed that the binding of sRNA and Hfq to the same site in mRNA is mutually exclusive (Beisel et al. 2012), and the MicC binding site overlaps with part of the ARN-5 motif (Fig. 1A). Hence, these results were not conclusive about the role of ARN sequences in Hfq binding and sRNA annealing to ompD mRNA.

To elucidate the importance of the SL2 region as a functional Hfq binding site, several adenosine residues in the ARN-3 motif and the following AU-rich sequence were substituted with other nucleotides, thus creating the ompD-187SL2mut construct (Table 4; Fig. 7A). The data showed that the rate of RybB annealing to this mRNA mutant was 200 -fold slower than to the wt $o m p D-187$, and 100 -fold slower than to the ompD-187-ARN-3 mutant (Table 4). Importantly, Hfq protein induced about a 200 -fold increase in the rate of RybB annealing to wt $o m p D-187$ as compared to only threefold for the ompD-187-SL2mut. This suggested the crucial importance of the AU-rich region for the RybB annealing. To confirm this conclusion, a shorter ompD-131 construct, containing $5^{\prime}$ terminal $131 \mathrm{nt}$, and its variants ompD-131-SL2mut, ompD-131 ARN-3, and ompD-131-AU were used (Fig. 7C,D,E). The rate of RybB annealing to ompD-131-SL2mut, which contained the same substitutions as ompD-187-SL2mut, was 50-fold slower than to wt ompD-131 and 30-fold slower than to ompD131 ARN-3 (Table 4). However, when only the AU-rich region was mutated (ompD-131-AU), the rate was similar to

TABLE 4. The sRNA annealing to $o m p D$ mRNA fragments with mutations in the SL2 region $^{\mathrm{a}}$

\begin{tabular}{|c|c|c|}
\hline \multirow[b]{2}{*}{ Interacting RNAs } & \multicolumn{2}{|c|}{$k_{\text {obs }}\left(\min ^{-1}\right)$} \\
\hline & $-\mathrm{Hfq}$ & $+\mathrm{Hfq}$ \\
\hline${ }^{32} \mathrm{P}-\mathrm{ompD}-187-\mathrm{RybB}$ & $0.032 \pm 0.005^{b}$ & $8.8 \pm 0.7^{b}$ \\
\hline${ }^{32} \mathrm{P}-\mathrm{ompD}-187$ ARN-3-RybB & $0.022 \pm 0.009^{c}$ & $3.2 \pm 1.1^{\mathrm{c}}$ \\
\hline${ }^{32} \mathrm{P}-\mathrm{ompD}-187-\mathrm{SL} 2 \mathrm{mut}-\mathrm{RybB}$ & $0.011 \pm 0.0018^{d}$ & $0.036 \pm 0.007^{d}$ \\
\hline${ }^{32} \mathrm{P}-\mathrm{ompD}-131-\mathrm{RybB}$ & $0.033 \pm 0.012$ & $2.5 \pm 0.44$ \\
\hline${ }^{32} \mathrm{P}-\mathrm{ompD}-131$ ARN-3-RybB & $0.022 \pm 0.0005$ & $1.8 \pm 0.41$ \\
\hline${ }^{32} \mathrm{P}-\mathrm{ompD}-131-\mathrm{SL} 2 \mathrm{mut}-\mathrm{RybB}$ & $0.021 \pm 0.0017$ & $0.046 \pm 0.012$ \\
\hline${ }^{32} \mathrm{P}-\mathrm{ompD}-131-\mathrm{AU}-\mathrm{RybB}$ & $0.019 \pm 0.0013$ & $0.17 \pm 0.08$ \\
\hline${ }^{32} \mathrm{P}-\mathrm{ompD}-187$-SdsR & $0.0029 \pm 0.0008^{b}$ & $0.01 \pm 0.0021^{b}$ \\
\hline${ }^{32} \mathrm{P}-\mathrm{ompD}-187$ ARN-3-SdsR & $<0.001$ & $0.005 \pm 0.0014$ \\
\hline${ }^{32} \mathrm{P}-\mathrm{ompD}-187-\mathrm{SL} 2 \mathrm{mut}-\mathrm{SdsR}$ & $<0.001^{\mathrm{d}}$ & $<0.001^{\mathrm{d}}$ \\
\hline${ }^{32} \mathrm{P}-\mathrm{ompD}-187$ - MicC & $0.025 \pm 0.0037^{b}$ & $6.9 \pm 0.79^{b}$ \\
\hline${ }^{32} \mathrm{P}-\mathrm{OmpD}-187$ ARN-3 - MicC & $0.019 \pm 0.0058^{\mathrm{c}}$ & $2.1 \pm 0.58^{\mathrm{c}}$ \\
\hline${ }^{32} \mathrm{P}-\mathrm{OmpD}-187-\mathrm{SL} 2 \mathrm{mut}-\mathrm{MicC}$ & $0.0088 \pm 0.002^{\mathrm{d}}$ & $0.032 \pm 0.0095^{\mathrm{d}}$ \\
\hline${ }^{32} \mathrm{P}-\mathrm{ompD}-187-\Delta \mathrm{SL} 2-\mathrm{MicC}$ & $0.023 \pm 0.0043$ & $0.9 \pm 0.27$ \\
\hline
\end{tabular}

The numbers are averages of at least three independent experiments.

${ }^{a}$ The concentration of ${ }^{32}$ P-labeled RNA was $1 \mathrm{nM}$, Hfq $3 \mathrm{nM}$; and unlabeled RNA $25 \mathrm{nM}$.

bValues from Table 2.

'Values from Supplemental Table S2.

dValues from Table 3. that for the ompD-131-SL2mut construct. This confirmed the important role of the AU-rich region for the Hfq-dependent annealing of RybB to ompD mRNA. Further analysis showed that the annealing of MicC and SdsR sRNAs was also detrimentally affected by the mutations in the ompD187-SL2mut construct (Table 4). The rate of MicC annealing to ompD-187-SL2mut was 200-fold slower than to wt ompD187 , and 70-fold slower than to ompD-187 ARN-3, while the rates of SdsR annealing to ompD-187-SL2mut were too slow to be accurately measured. The removal of the whole SL2 region in the ompD-187- $\Delta$ SL2 construct also negatively affected the MicC annealing but its effect was smaller than that of the mutations in ompD-187-SL2mut (Table 4; Fig. 7B). Overall, these data suggested that the long AU-rich sequence in the $5^{\prime}$-untranslated region of ompD is important for Hfqdependent annealing of all three sRNAs to the coding sequence of this mRNA.

To better understand how Hfq interacts with the SL2 region, the equilibrium binding of $\mathrm{Hfq}$ mutants to the ompD-187-SL2mut construct and their role in the sRNA annealing to this mRNA were analyzed (Tables 1,3 ). The affinity of the ompD-187-SL2mut construct to Hfq was about threefold weaker than that of wt ompD-187, and about twofold weaker than that of ompD-187 ARN-3, which contained only the mutations in the ARN-3 motif (Table 1). The affinity of Hfq with mutations Y25D and K56A to ompD-187SL2mut or ompD-187 ARN-3 was similar to that of wt ompD-187. However, the R16A Hfq mutant bound sixfold weaker to $o m p D-187 \mathrm{ARN}-3$, and 10-fold weaker to ompD$187-S L 2 m u t$ than to wt ompD-187. This suggests that contacts with the nonspecific rim binding site of $\mathrm{Hfq}$ compensate for the lost interactions with the distal face of Hfq in mRNAs that contain mutations in the ARN-3 motif and the AUrich region. To further investigate how Hfq interacts with the AU-rich region, the rates of RybB annealing to ompD187-SL2mut were compared in the presence of the Y25D, K56A, and R16A mutants of Hfq (Table 3). Even though RybB annealing to ompD-187-SL2mut was

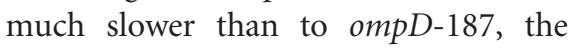
proximal and distal face mutations had the same detrimental role in RybB annealing, while the rim site did not affect it. The same mutations also had similar effects on the MicC annealing to ompD187-SL2mut, while the annealing of SdsR to this mRNA construct was too slow to accurately measure (Table 3 ). This suggested that the residual Hfq-dependent annealing of $\mathrm{RybB}$ and $\mathrm{MicC}$ sRNAs to ompD-187-SL2mut depended on the remaining contacts with the distal face of Hfq, and not on the alternative 
A

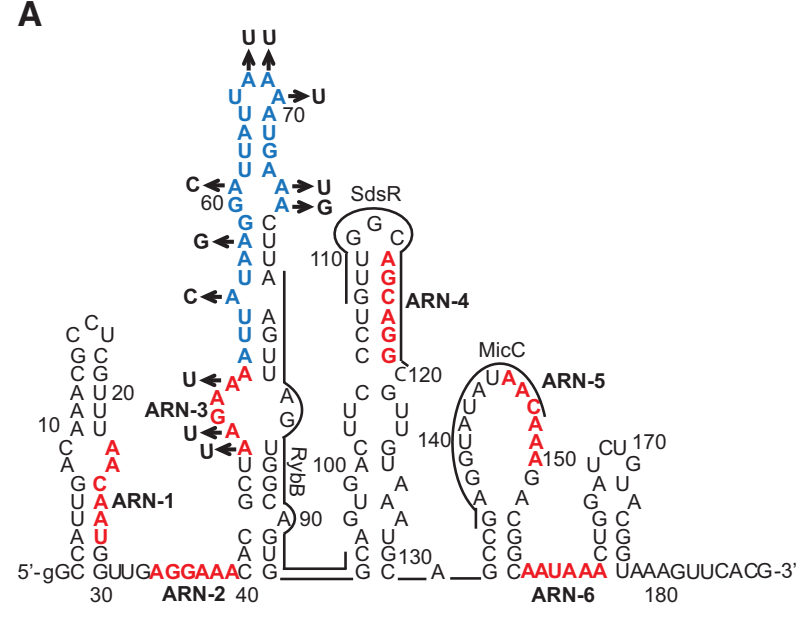

B

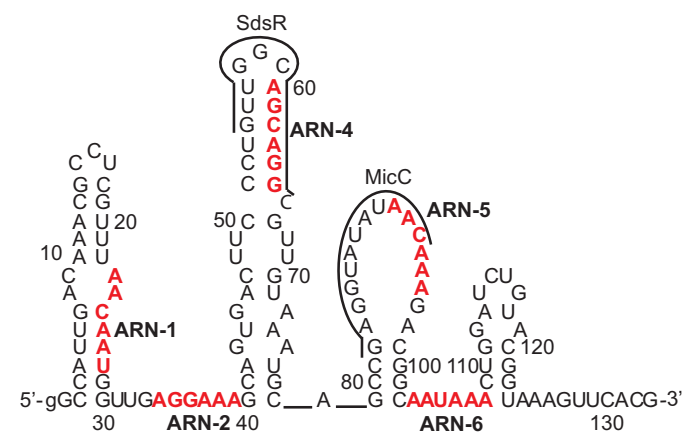

C

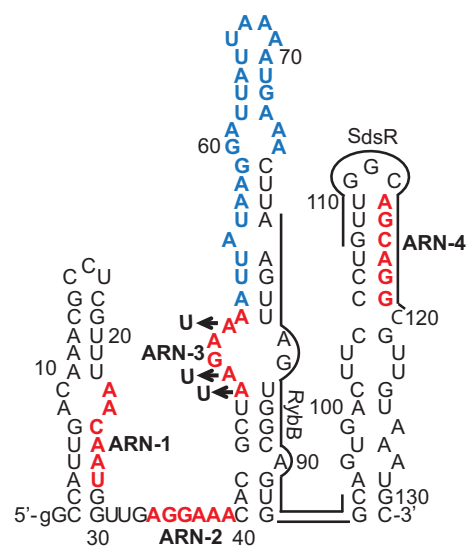

E

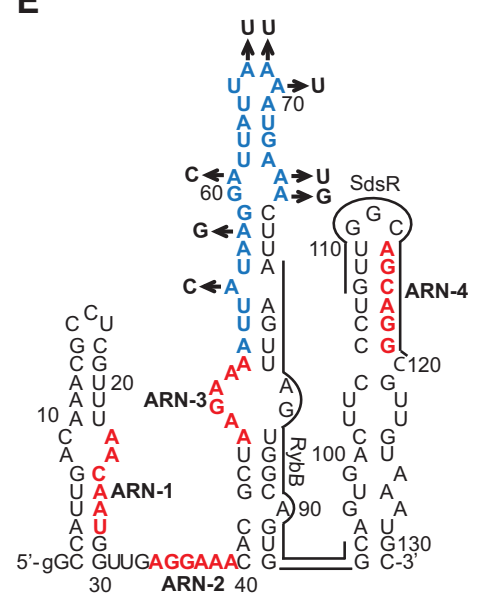

FIGURE 7. The nucleotide substitutions in SL2 region presented on the structure of ompD-187 or ompD-131. Point mutations used to generate $(A)$ ompD-187-SL2mut, $(B)$ ompD-187- $\Delta$ SL2, $(C)$ ompD-131 ARN3, $(D)$ ompD-131-SL2mut, $(E)$ ompD-131-AU mRNA mutants. The (ARN) ${ }_{2}$ motifs and the AU-rich sequence are marked in red and blue, respectively.

interactions with Hfq. Overall, these data indicated the essential role of the Hfq distal face contacts with the long AU-rich sequence in $\operatorname{ompD} \mathrm{mRNA}$ for the sRNA annealing.

\section{DISCUSSION}

The binding of Hfq to the $5^{\prime}$-untranslated region of $o m p D$ mRNA allows it to increase the rates of annealing of RybB, SdsR, and MicC sRNAs to the coding sequence (Table 2; Fig. 3). The Hfq-dependent acceleration of sRNA annealing to the mRNA untranslated region has been previously observed, for example, for OxyS annealing to fhlA mRNA (Zhang et al. 2002; Salim and Feig 2010), Spot42 to galK mRNA (Moller et al. 2002), and RNA-OUT to RNA-IN (Ross et al. 2013). However, the contribution of Hfq has been explored in molecular detail only for DsrA sRNA annealing to the $5^{\prime}$-untranslated region of $r p o S$ mRNA (Soper and Woodson 2008; Soper et al. 2011; Panja and Woodson 2012; Peng et al. 2014a,b). Hfq had about a 60-fold effect on the $k_{\mathrm{obs}}$ rate of DsrA annealing to rpoS mRNA (Peng et al. 2014b), and about a 20-fold on the rate of RNA-OUT annealing to RNA-IN (Ross et al. 2013). The data presented here showed that Hfq increased the rates of RybB and MicC annealing to the coding sequence of ompD mRNA about 250fold, and it also had a small effect on the rate of SdsR annealing (Table 2). Hence, similarly to those sRNAs, which bind to the untranslated regions, also sRNAs that bind to the coding sequence can use Hfq to increase their rates of annealing to the target mRNAs.

The Hfq protein contributed differently to the annealing of each of the three sRNAs to ompD mRNA (Table 2; Supplemental Fig. S3). The role of Hfq in the annealing of RybB to ompD mRNA was to overcome the energetic barriers formed by the structures of both interacting RNAs (Table 2). Hence, it was similar to the Hfq contribution to the annealing of DsrA sRNA, which involved mainly the rearrangement of rpoS mRNA structure, but was also partly dependent on the structure of DsrA (Soper et al. 2011). The contribution of $\mathrm{Hfq}$ to the annealing of SdsR was also dependent on the structures of both SdsR sRNA and ompD (Table 2; Supplemental 
Fig. S3). However, the Hfq-dependent increase of the SdsR annealing rate was much lower than for the other two sRNAs. In contrast, the structure of MicC sRNA appeared to be the crucial barrier to its annealing to $\operatorname{ompD}$ (Table 2; Supplemental Fig. S3). The minimal MicC sRNA in the absence of Hfq bound to ompD mRNA as fast as the full-length $\mathrm{MicC}$ in the presence of Hfq. This indicated that the main contribution of $\mathrm{Hfq}$ to the MicC annealing involved the rearrangement of the MicC structure or the recruitment of full length MicC towards its binding site in mRNA. These data suggested that the contributions of Hfq to each sRNA annealing to mRNA are individually adjusted depending on the structures of the interacting RNAs.

The long AU-rich sequence in the $5^{\prime}$-UTR served as the functionally important Hfq binding site in ompD mRNA (Tables 3, 4; Figs. 6, 7). This sequence consists of an $(\mathrm{AAN})_{2}$ repeat, which was named here as ARN-3, followed by a 24-nt long region, which contains adenosines at each third position making it an (ANN) ${ }_{8}$ motif (Fig. 1). Overall, this whole 30-nt long sequence contains 18 adenosines and eight uridines. The Hfq binding sites identified previously in mRNAs included both the ARN repeats (Soper and Woodson 2008; Link et al. 2009; Beisel et al. 2012) and the AU-rich regions (Schu et al. 2015; Updegrove et al. 2016). However, it was recently proposed that there is a continuum of possible interactions of Hfq with different RNA sequences (Schu et al. 2015). Consistently, it was also reported that Hfq binding sites in E. coli transcriptome often contained mismatches in ARN repeats (Tree et al. 2014). The dependence of RybB, SdsR, and MicC binding on the proximal face of Hfq (Table 1) is typical for Class I sRNAs (Zhang et al. 2013; Schu et al. 2015). This suggests that Hfq could use its unoccupied distal surface to bind $o m p D$ mRNA, when matching these sRNAs to their binding sites in ompD mRNA. Indeed, the annealing of RybB and MicC sRNAs to $o m p D$ was strongly detrimentally affected by the mutations in the proximal and distal face of Hfq, while the effect of the mutation in the rim was smaller (Table 3). In agreement with that, the location of adenosines in each third position of the AU-rich region is consistent with the distance between the adenosine specific binding pockets on the distal surface of Hfq (Link et al. 2009). Regardless of the detailed mechanism of $\mathrm{Hfq}$ binding to the AU-rich region of ompD mRNA, these data support the view that Hfq can use different binding modes to bring together the pairing regions of mRNA and sRNA molecules.

The rearrangements of mRNA structure are an important part of translation regulation mechanisms dependent on sRNAs. The Hfq protein rearranges the structure of rpoS mRNA to facilitate the annealing of DsrA sRNA (Soper et al. 2011), and the structure of $\operatorname{sodB}$ mRNA to promote the annealing of RyhB sRNA (Geissmann and Touati 2004). The mRNA structure rearrangements can also be induced by sRNAs. This is most evident for the three sRNAs that bind to the $5^{\prime}$-UTR of rpoS mRNA and change the equi- librium between ribosome-accessible and inaccessible conformations of this mRNA (Soper et al. 2010). The data presented here showed that the sRNA binding can also induce conformational changes in the $o m p D$ mRNA (Fig. 5). The binding of RybB sRNA induced rearrangements within $5^{\prime}$-UTR and at the beginning of the ompD coding sequence (Fig. 5A,C), while MicC induced local rearrangements in the coding sequence, which were consistent with $\mathrm{MicC}$ pairing to the large 16-nt loop of SL4 and the unfolding of the stem of SL4 (Fig. 5B,D). The sequence directly $3^{\prime}$ adjacent to the MicC binding site, which unfolding was detected by increased nuclease S1 cleavage (Fig. 5B), coincides with the region of increased RNase E cleavage in vivo (Pfeiffer et al. 2009), which may reflect the increased structural dynamics of this region upon $\mathrm{MicC}$ annealing. In another recent example, the conformational change induced by the annealing of MicF sRNA to the coding sequence of $l p x R$ mRNA resulted in increased susceptibility to cleavage by RNase E (Corcoran et al. 2012). A different outcome followed the binding of SR1 sRNA to the coding sequence of $a h r C$ mRNA of B. subtilis, which induced changes in the mRNA structure resulting in the repression of the initiation of translation (Heidrich et al. 2007).

Hfq promotes the annealing of sRNAs, which use different mechanisms in the regulation of ompD mRNA translation in vivo. Among the three sRNAs studied here, RybB binds at the beginning of the $o m p D$ coding sequence within the five-codon window, and interferes with the initiation of translation (Bouvier et al. 2008; Balbontin et al. 2010; Papenfort et al. 2010). SdsR anneals to ompD mRNA outside of the footprint of the initiating ribosome and is expected to affect mRNA decay rather than translation initiation (Frohlich et al. 2012). In contrast, the mode of action of $\mathrm{MicC}$ was proposed to involve the recruitment of RNase $\mathrm{E}$ to its binding site in mRNA leading to the accelerated decay of $o m p D$ mRNA (Pfeiffer et al. 2009; Bandyra et al. 2012). The fact that Hfa facilitates the annealing of sRNAs acting differently on translation (Table 2; Fig. 3) suggests that it has a general role in promoting the sRNA-mRNA pairing, regardless of the downstream effects of sRNA binding. Indeed, the same Hfq binding site in the $5^{\prime}$-UTR of ompD mRNA is used to promote the annealing of sRNAs RybB and MicC, which have different locations within the coding sequence and different mechanisms of action. However, it is also possible that the binding of Hfq to the downstream site within the coding sequence of ompD, which is not essential for sRNA annealing, could play distinct roles, such as the recruitment of RNase E, in this way affecting the regulation.

In summary, the data presented here show that a long AU-rich sequence in the $5^{\prime}$-untranslated region of the ompD mRNA served as an Hfq binding site essential for the accelerated annealing of sRNAs to its coding sequence. The data suggest that the contributions of Hfq to the annealing of each sRNA to the complementary region in ompD mRNA were individually tuned depending on the structures 
of sRNAs and the structural contexts of their binding sites in mRNA. These results support the view of Hfq as a generic multifaceted binder of RNA molecules, the versatile roles of which in RNA metabolism are specified by the properties of interacting RNA molecules.

\section{MATERIALS AND METHODS}

\section{RNA preparation}

sRNAs and fragments of $o m p D$ mRNA were synthesized using T7 RNA polymerase (Milligan et al. 1987). The templates for the in vitro transcription of wt sRNAs and their mutants and fragments of ompD mRNA shorter than $140 \mathrm{nt}$ were obtained by Taq polymerase extension of chemically synthesized overlapping oligodeoxyribonucleotides (oligo.pl) (Supplemental Table S3). The templates for the transcription of the longer fragments of $o m p D$ mRNA were obtained by PCR amplification from a pGEM T-Easy plasmid (Promega) containing a DNA sequence corresponding to nucleotides -103 to +210 of ompD mRNA from Salmonella typhimurium. To obtain templates for synthesis of mutated ompD mRNA fragments, the QuikChange Site-Directed Mutagenesis Kit (Stratagene) and specific primers (Supplemental Table S3) were used to introduce mutations into the $o m p D$ sequence in the pGEM T-Easy plasmid. After transcription the RNA molecules were purified using denaturing gel electrophoresis as previously described (Olejniczak 2011). RNAs were $5^{\prime}-{ }^{32} \mathrm{P}$-labeled using T4 polynucleotide kinase or $3^{\prime}$ end labeled with RNA ligase, which was followed by phenol-chloroform extraction, gel purification, and ethanol precipitation.

Chemically synthesized oligoribonucleotides SdsR-18 (sequence: GCCUGCAUUAAUGCCAAC), MicC-12 (sequence: GUUAUAU GCCUU), ompD-19S (sequence: CCUGUUGGCAGCAGGCGUU), and $o m p D-18 \mathrm{M}$ (sequence: GCCGAGGUAUAUAACAAA) were kind gifts of Professor Ryszard Kierzek (Institute of Bioorganic Chemistry of the Polish Academy of Sciences). The oligoribonucleotides RybB-16 (sequence: GCCACUGCUUUUCUUU) and ompD-21R (sequence: UAAGUUAGUGGCAGUGGCAGU) were purchased from Metabion International AG.

\section{Hfq protein purification}

The Salmonella Hfq protein with $\mathrm{His}_{6}$-tag on C-terminus was expressed from a pET15b vector (Novagen), in which the Hfq sequence was cloned via $\mathrm{NcoI}$ and BamHI restriction sites. This plasmid was used as a template for the preparation of Hfq R16A, Hfq Y25D, and $\mathrm{Hfq}$ K56A mutants using QuikChange Site-Directed Mutagenesis Kit (Stratagene) and specific primers (Supplemental Table S3). All constructs were verified by sequencing. Wt Hfq and its mutants were overexpressed and purified as described for E. coli Hfq (Małecka et al. 2015). The Hfq concentration was determined from absorption at $280 \mathrm{~nm}$ as previously described (Olejniczak 2011).

\section{Equilibrium binding assays}

To monitor the equilibrium binding of ${ }^{32} \mathrm{P}$-labeled RNAs to the Hfq protein, a gel shift assay was used. Reactions were carried out in $1 \times$ binding buffer (Lease and Woodson 2004) supplemented with $2 \mathrm{mM} \mathrm{MgCl}_{2}$ (24 mM Tris- $\mathrm{HCl} \mathrm{pH} 7.5,50 \mathrm{mM} \mathrm{NaCl}, 50 \mathrm{mM}$
$\mathrm{NH}_{4} \mathrm{Cl}, 50 \mathrm{mM} \mathrm{KCl}, 0.5 \mathrm{mM}$ EDTA, $2 \mathrm{mM} \mathrm{MgCl}_{2}$, and $5 \%$ glycerol) at room temperature. Prior to use, RNAs were heated for $1 \mathrm{~min}$ at $90^{\circ} \mathrm{C}$ followed by cooling for $10 \mathrm{~min}$ at room temperature.

The equilibrium binding reactions were prepared by mixing $15 \mu \mathrm{L}$ of $5^{\prime}-{ }^{32} \mathrm{P}$-labeled RNA ( $0.02 \mathrm{nM}$ concentration) with $15 \mu \mathrm{L}$ of $\mathrm{Hfq}$ dilutions for $1 \mathrm{~h}$ at RT. Twenty microliters of each sample was loaded on a $6 \%$ native polyacrylamide gel in $1 \times$ THEM2 $(66 \mathrm{mM}$ HEPES, $34 \mathrm{mM}$ Tris, $0.1 \mathrm{mM}$ EDTA, $2 \mathrm{mM} \mathrm{MgCl}_{2}$ ) (Peng et al. 2014b). Gels were dried, exposed to phoshor screens, and visualized using Fujifilm phosphorimager (FLA 5000) with MultiGauge software. The fraction bound in individual complexes was calculated as a proportion of the total counts in each lane. When one complex of RNA with Hfq was formed, data were fit to the Michaelis-Menten binding isotherm. When two RNA complexes with Hfq were formed, the data were fit to a partition function assuming two unequal independent binding sites, and when three complexes were formed, the data were fit to a partition function assuming one specific and two equal unspecific sites (Soper et al. 2011).

\section{Kinetics of sRNA-mRNA annealing}

The kinetics of RNA annealing was monitored by native mobility gel shift assays. RNAs were prepared as described above. All binding reactions were carried out at RT in $64 \mu \mathrm{L}$ volume in $1 \times$ binding buffer. In the reactions, $1 \mathrm{nM}^{32} \mathrm{P}$-labeled RNA was mixed with $25 \mathrm{nM}$ RNA in the presence or absence of $3 \mathrm{nM}$ Hfq hexamer. Native $6 \%$ polyacrylamide gels in $1 \times$ THEM2 were run continuously and aliquots were loaded onto the gel at specific time points $(0.5-60 \mathrm{~min})$. Controls were prepared in the same way as reactions, except that in the control reaction containing ompD-187 mRNA with Hfq the $2 \mathrm{nM}$ concentration of $0 m p D-187$ was added to $1 \mathrm{nM}{ }^{32} \mathrm{P}$-labeled ompD-187 to keep the concentration of RNA the same as the concentration of Hfq to prevent RNA-Hfq complex retention in the wells. Controls were loaded before the first time point or after the last time point. After electrophoresis, gels were treated as described above. The fraction bound of individual complexes was calculated as a proportion of the total counts in each lane (RNA in complexes and free RNA). The fractions of sRNA-mRNA or sRNA-mRNA-Hfq complexes were plotted versus time (0.5-60 min). Observed association rate $\left(k_{\mathrm{obs}}\right)$ values were calculated by fitting data to the singleexponential or double-exponential association equation, as described. For those reactions that were not complete at the last timepoint, the endpoint value of $70 \%$ was assumed in the data fitting.

\section{In vitro structure probing, footprinting, and boundary experiments}

In vitro structure probing reactions were performed on $5^{\prime}$ end-labeled RNAs in a total volume of $10 \mu \mathrm{L}$. The RNA was denatured at $90^{\circ} \mathrm{C}$ for $1 \mathrm{~min}$ followed by incubation at room temperature for $10 \mathrm{~min}$. Secondary structure probing with RNase T1 was performed in $12 \mathrm{mM}$ Tris- $\mathrm{HCl}$, pH 7.2, $48 \mathrm{mM} \mathrm{NaCl}$, and $1.2 \mathrm{mM} \mathrm{MgCl}_{2}$ at RT for $10 \mathrm{~min}$. RNase T2 digestion was carried out in $10 \mathrm{mM}$ Tris $\mathrm{pH} 7$, $100 \mathrm{mM} \mathrm{KCl}$, and $10 \mathrm{mM} \mathrm{MgCl}_{2}$. RNase T2 reactions contained $1 \mu \mathrm{g}$ of total yeast RNA (Ambion) and were incubated at RT for $15 \mathrm{~min}$. To map nuclease S1 cleavage sites, RNA was incubated in $40 \mathrm{mM}$ sodium acetate $\mathrm{pH} 4.5,300 \mathrm{mM} \mathrm{NaCl}$, and $2 \mathrm{mM} \mathrm{ZnSO}_{4}$ at RT for $10 \mathrm{~min}$. RNase III digestions were performed in the same buffer as RNase T2 digestions, except for the addition of 
$1 \mathrm{mM}$ DTT. Reactions were incubated at $37^{\circ} \mathrm{C}$ for $5 \mathrm{~min}$. Reactions with RNase T1, T2, S1, and RNase III were quenched by addition of $10 \mu \mathrm{L}$ of stop buffer ( $8 \mathrm{M}$ urea and $20 \mathrm{mM}$ EDTA). Formamide ladder was obtained by incubating ${ }^{32} \mathrm{P}$-labeled RNA in formamide (1:5 proportion) at $100^{\circ} \mathrm{C}$ for $1 \mathrm{~h}$. Reaction was stopped by cooling on ice. To obtain RNase T1 ladder, ${ }^{32} \mathrm{P}$-labeled RNA was incubated in $50 \mathrm{mM}$ sodium citrate $(\mathrm{pH} 4.3)$ and $7 \mathrm{M}$ urea at $55^{\circ} \mathrm{C}$ for $10 \mathrm{~min}$. Reaction was quenched by adding $10 \mu \mathrm{L}$ of stop buffer. Samples were loaded on a 10\% PAGE. Gels were frozen and exposed to phosphor screens overnight. Data were quantified using PhosphorImager.

To monitor changes in RNase accessibility of ompD mRNA structure upon sRNA binding, $5^{\prime}$ end-labeled $o m p D-187$ or $5^{\prime}$ end-labeled ompD-93-187 were prepared as described above and then mixed with appropriate sRNA concentration in S1 reaction buffer. After 20 min of incubation, $1 \mu \mathrm{L}$ of nuclease S1 (2U) was added to each reaction. After incubation at RT for $10 \mathrm{~min}$, reactions were stopped and processed as described above.

To determine the location of $\mathrm{Hfq}$ binding sites within ompD mRNA leader, boundary experiments were performed. $5^{\prime}$ end-labeled or $3^{\prime}$ end-labeled $o m p D-187$ molecules were prepared as described above and then subjected to RNase T1 or nuclease S1 cleavage in $\mathrm{T} 1$ or $\mathrm{S} 1$ reaction buffer, respectively. Reactions were incubated at RT for $10 \mathrm{~min}$ and stopped by addition of EDTA to the final concentration of $10 \mathrm{mM}$. Then, reactions were mixed with appropriate $\mathrm{Hfq}$ concentration. After $20 \mathrm{~min}$ of incubation at RT, 10 $\mu \mathrm{L}$ of $8 \mathrm{M}$ urea was added to each tube, reaction products were resolved on $10 \%$ polyacrylamide gel in denaturing conditions, and analyzed as described above.

\section{SUPPLEMENTAL MATERIAL}

Supplemental material is available for this article.

\section{ACKNOWLEDGMENTS}

We thank Olke Uhlenbeck, Sarah Woodson, Jörg Vogel, and Sarah Ledoux for helpful discussions, and S.L. for critical comments on the manuscript. This work was supported by the National Science Centre in Poland (grant no. 2011/01/B/NZ1/05325), KNOW RNA Research Centre in Poznań (no. 01/KNOW2/2014), and the Foundation for Polish Science (grant no. TEAM/2011-8/5) cofinanced by the European Union Regional Development Fund within the framework of the Operational Program Innovative Economy.

Received November 21, 2015; accepted April 1, 2016.

\section{REFERENCES}

Andrade JM, Pobre V, Matos AM, Arraiano CM. 2012. The crucial role of PNPase in the degradation of small RNAs that are not associated with Hfq. RNA 18: 844-855.

Balbontin R, Fiorini F, Figueroa-Bossi N, Casadesus J, Bossi L. 2010. Recognition of heptameric seed sequence underlies multi-target regulation by RybB small RNA in Salmonella enterica. Mol Microbiol 78: 380-394.

Bandyra KJ, Said N, Pfeiffer V, Gorna MW, Vogel J, Luisi BF. 2012. The seed region of a small RNA drives the controlled destruction of the target mRNA by the endoribonuclease RNase E. Mol Cell 47: 943-953.
Beisel CL, Updegrove TB, Janson BJ, Storz G. 2012. Multiple factors dictate target selection by Hfq-binding small RNAs. EMBO $J$ 31: 1961-1974.

Bobrovskyy M, Vanderpool CK. 2016. Diverse mechanisms of posttranscriptional repression by the small RNA regulator of glucosephosphate stress. Mol Microbiol 99: 254-273.

Bossi L, Maloriol D, Figueroa-Bossi N. 2008. Porin biogenesis activates the $\sigma^{\mathrm{E}}$ response in Salmonella hfq mutants. Biochimie 90: 1539-1544.

Bouvier M, Sharma CM, Mika F, Nierhaus KH, Vogel J. 2008. Small RNA binding to $5^{\prime}$ mRNA coding region inhibits translational initiation. Mol Cell 32: 827-837.

Brescia CC, Mikulecky PJ, Feig AL, Sledjeski DD. 2003. Identification of the Hfq-binding site on DsrA RNA: Hfq binds without altering DsrA secondary structure. RNA 9: 33-43.

Corcoran CP, Podkaminski D, Papenfort K, Urban JH, Hinton JC, Vogel J. 2012. Superfolder GFP reporters validate diverse new mRNA targets of the classic porin regulator, MicF RNA. Mol Microbiol 84: 428-445.

de Haseth PL, Uhlenbeck OC. 1980. Interaction of Escherichia coli host factor protein with oligoriboadenylates. Biochemistry 19: 6138-6146.

De Lay N, Gottesman S. 2012. A complex network of small non-coding RNAs regulate motility in Escherichia coli. Mol Microbiol 86: 524-538.

Desnoyers G, Masse E. 2012. Noncanonical repression of translation initiation through small RNA recruitment of the RNA chaperone Hfq. Genes Dev 26: 726-739.

Ellis MJ, Trussler RS, Haniford DB. 2015. Hfq binds directly to the ribosome-binding site of IS 10 transposase mRNA to inhibit translation. Mol Microbiol 96: 633-650.

Fei J, Singh D, Zhang Q, Park S, Balasubramanian D, Golding I, Vanderpool CK, Ha T. 2015. RNA biochemistry. Determination of in vivo target search kinetics of regulatory noncoding RNA. Science 347: 1371-1374.

Frohlich KS, Papenfort K, Berger AA, Vogel J. 2012. A conserved RpoSdependent small RNA controls the synthesis of major porin OmpD. Nucleic Acids Res 40: 3623-3640.

Geissmann TA, Touati D. 2004. Hfq, a new chaperoning role: binding to messenger RNA determines access for small RNA regulator. EMBO J 23: 396-405.

Guo MS, Updegrove TB, Gogol EB, Shabalina SA, Gross CA, Storz G. 2014. MicL, a new $\sigma^{\mathrm{E}}$-dependent sRNA, combats envelope stress by repressing synthesis of Lpp, the major outer membrane lipoprotein. Genes Dev 28: 1620-1634.

Gutierrez A, Laureti L, Crussard S, Abida H, Rodriguez-Rojas A, Blazquez J, Baharoglu Z, Mazel D, Darfeuille F, Vogel J, et al. 2013. $\beta$-Lactam antibiotics promote bacterial mutagenesis via an RpoS-mediated reduction in replication fidelity. Nat Commun 4: 1610.

Halder S, Bhattacharyya D. 2013. RNA structure and dynamics: a base pairing perspective. Prog Biophys Mol Biol 113: 264-283.

Heidrich N, Moll I, Brantl S. 2007. In vitro analysis of the interaction between the small RNA SR1 and its primary target ahrC mRNA. Nucleic Acids Res 35: 4331-4346.

Ikeda Y, Yagi M, Morita T, Aiba H. 2011. Hfq binding at RhlB-recognition region of RNase $\mathrm{E}$ is crucial for the rapid degradation of target mRNAs mediated by sRNAs in Escherichia coli. Mol Microbiol 79: 419-432.

Kovach AR, Hoff KE, Canty JT, Orans J, Brennan RG. 2014. Recognition of U-rich RNA by Hfq from the Gram-positive pathogen Listeria monocytogenes. RNA 20: 1548-1559.

Lease RA, Woodson SA. 2004. Cycling of the Sm-like protein Hfq on the DsrA small regulatory RNA. J Mol Biol 344: 1211-1223.

Link TM, Valentin-Hansen P, Brennan RG. 2009. Structure of Escherichia coli $\mathrm{Hfq}$ bound to polyriboadenylate RNA. Proc Natl Acad Sci 106: 19292-19297.

Maki K, Uno K, Morita T, Aiba H. 2008. RNA, but not protein partners, is directly responsible for translational silencing by a bacterial Hfqbinding small RNA. Proc Natl Acad Sci 105: 10332-10337. 
Małecka EM, Stróżecka J, Sobańska D, Olejniczak M. 2015. Structure of bacterial regulatory RNAs determines their performance in competition for the chaperone protein Hfq. Biochemistry 54: 1157-1170.

Mikulecky PJ, Kaw MK, Brescia CC, Takach JC, Sledjeski DD, Feig AL. 2004. Escherichia coli Hfq has distinct interaction surfaces for DsrA, rpoS and poly(A) RNAs. Nat Struct Mol Biol 11: 1206-1214.

Milligan JF, Groebe DR, Witherell GW, Uhlenbeck OC. 1987. Oligoribonucleotide synthesis using T7 RNA polymerase and synthetic DNA templates. Nucleic Acids Res 15: 8783-8798.

Moller T, Franch T, Hojrup P, Keene DR, Bachinger HP, Brennan RG, Valentin-Hansen P. 2002. Hfq: a bacterial Sm-like protein that mediates RNA-RNA interaction. Mol Cell 9: 23-30.

Olejniczak M. 2011. Despite similar binding to the Hfq protein regulatory RNAs widely differ in their competition performance. Biochemistry 50: 4427-4440.

Panja S, Woodson SA. 2012. Hfq proximity and orientation controls RNA annealing. Nucleic Acids Res 40: 8690-8697.

Panja S, Santiago-Frangos A, Schu DJ, Gottesman S, Woodson SA. 2015. Acidic residues in the Hfq chaperone increase the selectivity of sRNA binding and annealing. J Mol Biol 427: 3491-3500.

Papenfort K, Vogel J. 2010. Regulatory RNA in bacterial pathogens. Cell Host Microbe 8: 116-127.

Papenfort K, Pfeiffer V, Mika F, Lucchini S, Hinton JC, Vogel J. 2006. $\sigma^{\mathrm{E}}$-dependent small RNAs of Salmonella respond to membrane stress by accelerating global omp mRNA decay. Mol Microbiol 62: $1674-1688$

Papenfort K, Bouvier M, Mika F, Sharma CM, Vogel J. 2010. Evidence for an autonomous $5^{\prime}$ target recognition domain in an Hfq-associated small RNA. Proc Natl Acad Sci 107: 20435-20440.

Papenfort K, Sun Y, Miyakoshi M, Vanderpool CK, Vogel J. 2013. Small RNA-mediated activation of sugar phosphatase mRNA regulates glucose homeostasis. Cell 153: 426-437.

Peng Y, Curtis JE, Fang X, Woodson SA. 2014a. Structural model of an mRNA in complex with the bacterial chaperone Hfq. Proc Natl Acad Sci 111: 17134-17139.

Peng Y, Soper TJ, Woodson SA. 2014b. Positional effects of AAN motifs in rpoS regulation by sRNAs and Hfq. J Mol Biol 426: 275-285.

Pfeiffer V, Sittka A, Tomer R, Tedin K, Brinkmann V, Vogel J. 2007. A small non-coding RNA of the invasion gene island (SPI-1) represses outer membrane protein synthesis from the Salmonella core genome. Mol Microbiol 66: 1174-1191.

Pfeiffer V, Papenfort K, Lucchini S, Hinton JC, Vogel J. 2009. Coding sequence targeting by MicC RNA reveals bacterial mRNA silencing downstream of translational initiation. Nat Struct Mol Biol 16: 840-846.

Qu X, Wen JD, Lancaster L, Noller HF, Bustamante C, Tinoco I Jr. 2011. The ribosome uses two active mechanisms to unwind messenger RNA during translation. Nature 475: 118-121.

Reuter JS, Mathews DH. 2010. RNAstructure: software for RNA secondary structure prediction and analysis. BMC Bioinformatics 11: 129.

Robinson KE, Orans J, Kovach AR, Link TM, Brennan RG. 2014. Mapping Hfq-RNA interaction surfaces using tryptophan fluorescence quenching. Nucleic Acids Res 42: 2736-2749.

Ross JA, Ellis MJ, Hossain S, Haniford DB. 2013. Hfq restructures RNAIN and RNA-OUT and facilitates antisense pairing in the Tn10/IS10 system. RNA 19: 670-684.
Salim NN, Feig AL. 2010. An upstream Hfq binding site in the fhlA mRNA leader region facilitates the OxyS-fhlA interaction. PLoS One 5: e13028.

Salim NN, Faner MA, Philip JA, Feig AL. 2012. Requirement of upstream Hfq-binding $(\mathrm{ARN})_{\mathrm{x}}$ elements in $g l m S$ and the Hfq C-terminal region for GlmS upregulation by sRNAs GlmZ and GlmY. Nucleic Acids Res 40: 8021-8032.

Salvail H, Caron MP, Belanger J, Masse E. 2013. Antagonistic functions between the RNA chaperone Hfq and an sRNA regulate sensitivity to the antibiotic colicin. EMBO J 32: 2764-2778.

Sauer E, Weichenrieder O. 2011. Structural basis for RNA 3 '-end recognition by Hfq. Proc Natl Acad Sci 108: 13065-13070.

Sauer E, Schmidt S, Weichenrieder O. 2012. Small RNA binding to the lateral surface of Hfq hexamers and structural rearrangements upon mRNA target recognition. Proc Natl Acad Sci 109: 9396-9401.

Schu DJ, Zhang A, Gottesman S, Storz G. 2015. Alternative Hfq-sRNA interaction modes dictate alternative mRNA recognition. EMBO J 34: 2557-2573.

Sittka A, Pfeiffer V, Tedin K, Vogel J. 2007. The RNA chaperone Hfq is essential for the virulence of Salmonella typhimurium. Mol Microbiol 63: 193-217.

Sittka A, Lucchini S, Papenfort K, Sharma CM, Rolle K, Binnewies TT, Hinton JC, Vogel J. 2008. Deep sequencing analysis of small noncoding RNA and mRNA targets of the global post-transcriptional regulator, Hfq. PLoS Genet 4: e1000163.

Sledjeski DD, Whitman C, Zhang A. 2001. Hfq is necessary for regulation by the untranslated RNA DsrA. J Bacteriol 183: 1997-2005.

Sonnleitner E, Blasi U. 2014. Regulation of Hfq by the RNA CrcZ in Pseudomonas aeruginosa carbon catabolite repression. PLoS Genet 10: e1004440.

Soper TJ, Woodson SA. 2008. The rpoS mRNA leader recruits Hfq to facilitate annealing with DsrA sRNA. RNA 14: 1907-1917.

Soper T, Mandin P, Majdalani N, Gottesman S, Woodson SA. 2010. Positive regulation by small RNAs and the role of Hfq. Proc Natl Acad Sci USA 107: 9602-9607.

Soper TJ, Doxzen K, Woodson SA. 2011. Major role for mRNA binding and restructuring in sRNA recruitment by Hfq. RNA 17: 1544-1550.

Takyar S, Hickerson RP, Noller HF. 2005. mRNA helicase activity of the ribosome. Cell 120: 49-58.

Tree JJ, Granneman S, McAteer SP, Tollervey D, Gally DL. 2014. Identification of bacteriophage-encoded anti-sRNAs in pathogenic Escherichia coli. Mol Cell 55: 199-213.

Updegrove TB, Shabalina SA, Storz G. 2015. How do base-pairing small RNAs evolve? FEMS Microbiol Rev 39: 379-391.

Updegrove TB, Zhang A, Storz G. 2016. Hfq: the flexible RNA matchmaker. Curr Opin Microbiol 30: 133-138.

Waters LS, Storz G. 2009. Regulatory RNAs in bacteria. Cell 136: 615-628.

Zhang A, Wassarman KM, Ortega J, Steven AC, Storz G. 2002. The Smlike Hfq protein increases OxyS RNA interaction with target mRNAs. Mol Cell 9: 11-22.

Zhang A, Schu DJ, Tjaden BC, Storz G, Gottesman S. 2013. Mutations in interaction surfaces differentially impact $E$. coli $\mathrm{Hfq}$ association with small RNAs and their mRNA targets. J Mol Biol 425: 3678-3697. 

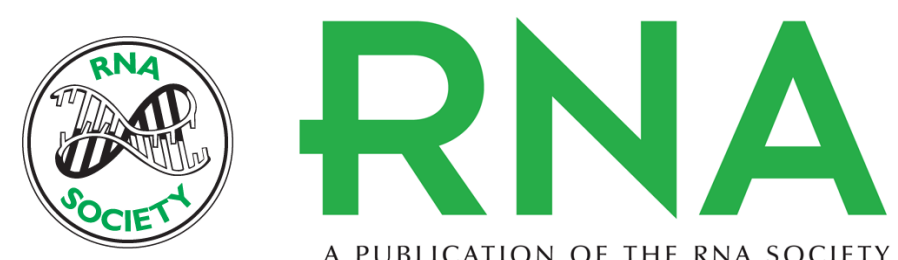

A PUBLICATION OF THE RNA SOCIETY

\section{Hfq assists small RNAs in binding to the coding sequence of $o m p D$ mRNA and in rearranging its structure}

Zuzanna Wroblewska and Mikolaj Olejniczak

RNA 2016 22: 979-994 originally published online May 6, 2016

Access the most recent version at doi:10.1261/rna.055251.115

\section{Supplemental http://rnajournal.cshlp.org/content/suppl/2016/05/05/rna.055251.115.DC1 Material}

References This article cites 64 articles, 21 of which can be accessed free at: http://rnajournal.cshlp.org/content/22/7/979.full.html\#ref-list-1

Open Access Freely available online through the RNA Open Access option.

Creative This article, published in $R N A$, is available under a Creative Commons License Commons (Attribution 4.0 International), as described at

License http://creativecommons.org/licenses/by/4.0/.

Email Alerting Receive free email alerts when new articles cite this article - sign up in the box at the Service top right corner of the article or click here.

To subscribe to $R N A$ go to:

http://rnajournal.cshlp.org/subscriptions 